\title{
The class II phosphoinositide 3-kinases PI3K-C2 $\alpha$ and PI3K-C2 $\beta$ differentially regulate clathrin-dependent pinocytosis in human vascular endothelial cells
}

\author{
Khin Thuzar Aung ${ }^{1} \cdot$ Kazuaki Yoshioka $^{1} \cdot$ Sho Aki ${ }^{1} \cdot$ Kazuhiro Ishimaru $^{1} \cdot$ Noriko Takuwa $^{1,2} \cdot$ Yoh Takuwa $^{1}$
}

Received: 20 August 2018 / Accepted: 13 October 2018 / Published online: 29 October 2018

(c) The Physiological Society of Japan and Springer Japan KK, part of Springer Nature 2018

\begin{abstract}
Pinocytosis is an important fundamental cellular process that is used by the cell to transport fluid and solutes. Phosphoinositide 3-kinases (PI3Ks) regulate a diverse array of dynamic membrane events. However, it is not well-understood which $\mathrm{PI} 3 \mathrm{~K}$ isoforms are involved in specific mechanisms of pinocytosis. We performed knockdown studies of endogenous PI3K isoforms and clathrin heavy chain (CHC) mediated by small interfering RNA (siRNA). The results demonstrated that the class II PI3K PI3K-C2 $\alpha$ and PI3K-C2 $\beta$, but not the class I or III PI3K, were required for pinocytosis, based on an evaluation of fluorescein-5-isothiocyanate (FITC)-dextran uptake in endothelial cells. Pinocytosis was partially dependent on both clathrin and dynamin, and both PI3K-C $2 \alpha$ and PI3K-C $2 \beta$ were required for clathrin-mediated-but not clathrin-nonmediated-FITC-dextran uptake at the step leading up to its delivery to early endosomes. Both PI3K-C $2 \alpha$ and PI3K-C $2 \beta$ were co-localized with clathrin-coated pits and vesicles. However, PI3K-C2 $\beta$, but not PI3K-C2 $\alpha$, was highly co-localized with actin filament-associated clathrin-coated structures and required for actin filament formation at the clathrin-coated structures. These results indicate that PI3K-C $2 \alpha$ and PI3K-C2 $\beta$ play differential, indispensable roles in clathrin-mediated pinocytosis.
\end{abstract}

Keywords Class II PI3K $\cdot$ PI3K-C2 $\alpha \cdot$ PI3K-C2 $\beta \cdot$ Pinocytosis $\cdot$ Clathrin $\cdot$ Endothelial cell

\section{Introduction}

Pinocytosis or fluid-phase endocytosis is an endocytic process in which extracellular fluid is engulfed into endocytic vesicles [1]. The known processes for endocytic vesicle formation are clathrin-dependent, caveolin-dependent and combined clathrin-/caveolin-independent processes [2, 3], among which clathrin-dependent endocytosis has been best characterized to date. Clathrin-dependent endocytosis begins with the formation of invaginations or pits at the plasma membrane, followed by the formation of endocytic

Electronic supplementary material The online version of this article (https://doi.org/10.1007/s12576-018-0644-2) contains supplementary material, which is available to authorized users.

Yoh Takuwa

ytakuwa@med.kanazawa-u.ac.jp

1 Department of Physiology, Kanazawa University School of Medicine, Kanazawa, Ishikawa 920-8640, Japan

2 Department of Health Science, Ishikawa Prefectural University, Kahoku, Ishikawa 929-1210, Japan vesicles through the pinching-off of the invagination neck by dynamin. In endocytic processes, a complex protein machinery works along with clathrins. The actin cytoskeletons are organized at clathrin-coated pits (actin patches) and participate in the growth of clathrin-coated pits. Pinocytosis takes place in almost all types of cells, but its role in blood capillary endothelial cells has been recognized as an important portal of trans-endothelial transport of proteins, lipids and lipoproteins, drugs and cell-secreted exosomes and microparticles [4].

Endocytic processes are regulated by polyphosphoinositides (PPIs) [5], small G proteins, including Rab [6], and other proteins [7]. PPIs are produced by phosphorylation of the inositol ring through the actions of phosphoinositide kinases. Among these, phosphoinositide 3-kinases (PI3K), which catalyze the phosphorylation of the D3 position within the inositol ring, play particularly important roles in membrane trafficking, including endocytosis. There are three classes of PI3K. Class I PI3Ks are activated mainly by receptor tyrosine kinases and $\mathrm{G}$ protein-coupled receptors to exert an effect on cell proliferation, migration and control of cell metabolism by generating $\mathrm{PI}(3,4) \mathrm{P}_{2}$ and $\mathrm{PI}(3,4,5) \mathrm{P}_{3}$ 
[8-10]. Class III PI3K, Vps34, stimulates the autophagic pathway by generating $\mathrm{PI}(3) \mathrm{P}[11,12]$. In contrast to class I and class III PI3K, the roles of class II PI3K, comprising $\mathrm{PI} 3 \mathrm{~K}-\mathrm{C} 2 \alpha,-\mathrm{C} 2 \beta$ and $-\mathrm{C} 2 \gamma$, remain poorly understood. However, recent studies, including those from our research group, have shown that PI3K-C2 $\alpha$ is required for the endocytosis of certain membrane proteins, such as vascular endothelial growth factor receptor and sphingosine-1-phosphate receptor, while PI3K-C2 $\beta$ is involved in the regulation of autophagy [13-17]. The molecular and cellular mechanisms of pinocytosis, including clathrin dependence or independence and the requirement of PI3K, are not fully understood.

Of the three members of class II PI3K, PI $3 \mathrm{~K}-\mathrm{C} 2 \alpha$ and PI3K-C2 $\beta$, are highly homologous and widely expressed, whereas PI3K-C $2 \gamma$ shows less homology to PI3K-C $2 \alpha$ and $\mathrm{PI} 3 \mathrm{~K}-\mathrm{C} 2 \beta$ and restricted expression in certain organs, including the liver and prostate $[18,19]$. Therefore, in the study reported here we addressed whether and how class II $\mathrm{PI} 3 \mathrm{~K}-\mathrm{C} 2 \alpha$ and $-\mathrm{C} 2 \beta$ are involved in pinocytosis in human vascular endothelial cells. We found that PI $3 \mathrm{~K}-\mathrm{C} 2 \alpha$ and $-\mathrm{C} 2 \beta$ were both required for clathrin-dependent pinocytosis but not for clathrin-independent pinocytosis. Interestingly, super-resolution microscopy revealed that both PI3K-C $2 \alpha$ and PI3K-C $2 \beta$ were co-localized to clathrin-coated pits and vesicles, but that only PI3K-C $2 \beta$ was highly co-localized to actin filament (F-actin)-associated clathrin-coated pits and vesicles. These observations suggest that PI $3 \mathrm{~K}-\mathrm{C} 2 \alpha$ and $\mathrm{PI} 3 \mathrm{~K}-\mathrm{C} 2 \beta$ are involved in clathrin-mediated pinocytosis through different mechanisms.

\section{Materials and methods}

\section{Cell culture, reagents and transfection}

Human umbilical vein endothelial cells (HUVECs) (cata$\log$ no. C2517A; Lonza, Basel, Switzerland) were plated on type-I collagen (Nitta Gelatin, Osaka, Japan)-coated dishes and cultured in endothelial growth medium (EGM-2) supplemented with $2 \%$ fetal bovine serum (FBS) and growth factor-enriched cocktails (catalog no. CC-3162; Lonza) at $37{ }^{\circ} \mathrm{C}$ under $5 \% \mathrm{CO}_{2}$. Cells between passage 4-6 were used for subsequent experiments. Chemical inhibitors used in this study were Pitstop-2 (catalog no. ab1206871; Abcam, Cambridge, UK), wortmannin (WMN) (catalog no. 681675; Calbiochem, Darmstadt, Germany), GDC-0941(Pictilisib) (catalog no. ab141352; Abcam) and 3-methyladenine (3-MA) (catalog no. S-2767, Selleck Chemicals, Japan). Knockdown of endogenous PI3K isoforms and clathrin heavy chain $(\mathrm{CHC})$ were performed with small interfering RNA (siRNA) transfection unless otherwise stated.

The siRNAs used in this study were synthesized using a Silencer siRNA construction kit (catalog no. AM1620;
Ambion, Austin, TX) according to the manufacturer's protocol. The targeted sequences of siRNA were:

\section{5'-AAGGUUGGCACUUACAAGAAU-3' for human PI3K-C2 $\alpha(P I K 3 C 2 A)$ \\ 5'-AAGCCGGAAGCUUCUGGGUUU-3' for human PI3K-C2 $\beta(P I K 3 C 2 B)$ \\ 5'-AAACUCAACACUGGCUAAUUA-3' for human Vps34 (PIK3C3) \\ 5'-GGACAACUGUUUCAUAUAG-3' for human PI3K p110 $\alpha(P I K 3 C A)$ \\ 5'-AAUCCAAUUCGAAGACCAAUU-3' for human CHC (CLTC).}

Intersectin1 (ITSN1) siRNAs was purchased from Invitrogen (catalog no.10620318; Invitrogen/Thermo Fisher Scientific, Carlsbad, CA, USA) and the targeted sequence was 5'-GGAAUCGAAGGCAAGAACUACUAAA-3'. The scrambled siRNA sequence was 5'-AAUUCUCCGAAC GUGUCACGU-3'. Cells were transiently transfected with specific siRNAs and Lipofectamine 2000 (catalog no.11668019; Invitrogen/Thermo Fisher Scientific) or Lipofectamine RNAiMAX (catalog no.13778075; Invitrogen/Thermo Fisher Scientific) in Opti-MEM (catalog no.31985070; Invitrogen/Thermo Fisher Scientific) according to the manufacturer's instruction for $48-72 \mathrm{~h}$ before the experiments. The single knockdown was conducted by transfecting cells with $50 \mathrm{nM}$ of siRNA, whereas the double knockdown was conducted by transfecting cells with the two specific siRNAs at $25 \mathrm{nM}$ each. The transfection efficiency of the siRNA, as evaluated with the fluorescent dye fluorescein amiditelabeled negative control (siRNA No.1; 5'-AGUACUGCU UACGAUACGGTT-3') (catalog no. AM4620; Ambion), was $94 \%$ in HUVECs. The efficiency of siRNA-mediated knockdown was confirmed by Western blotting using specific antibodies.

The enhanced green fluorescent protein (GFP)-tagged PI3K-C2 $\alpha$ (GFP-C2 $\alpha$ ) expression vector was as described previously [13]. PI3K-C2 $\beta$ complimentary DNA (cDNA) was obtained from K. Kitatani (Setsunan University, Osaka) [20]. For the expression vector for mCherry-tagged PI3K$\mathrm{C} 2 \alpha$ (mCherry-C2 $\alpha$ ) and GFP- or mCherry-tagged PI3KC $2 \beta$ (GFP-C $2 \beta$, mCherry-C2 $\beta$ ), human PIK $3 C 2 A$ and $P I K 3 C 2 B$ cDNA fragments were amplified by PCR using PrimeSTAR HS DNA Polymerase (catalog no. R010A; Takara-Bio Co., Shiga, Japan) according to the manufacturer's protocol. Sub-cloning of the PCR products into pmCherry-C1 (catalog no. 632524; Clontech-Takara Bio Inc., Shiga, Japan) and pAcGFP1-N vectors (catalog no. 632501; Clontech Laboratories, Mountain View, CA, USA) was performed using the In-Fusion HD Cloning kit (catalog no. Z9633 N; Clontech Laboratories). The monomeric red fluorescent protein (mRFP)-tagged wild-type 
dynamin-2 (WT-dynamin2) and dominant negative K44A mutant of dynamin-2 (K44A-dynamin2) were kindly provided by Dr. Pietro De Camilli, Yale University School of Medicine. The mEmerald-Lifeact-7 plasmid was a gift from Michael Davidson (Addgene plasmid \#54148 and \#56249, respectively). The mRFP-clathrin light chain (CLC) was a gift from Ari Helenius (Addgene plasmid \#14435). The GFP-intersectin1 short-isoform (ITSN1) was a gift from Peter McPherson (Addgene plasmid \#47394). All plasmids were purified using the Endotoxin-Free Plasmid Maxi Kit (catalog no. 12362; Qiagen, Hilden, Germany). Cells were transfected with expression plasmids using the NucleofactorI electroporation device (Program A-034; Lonza) with the Amaxa Nucleofector kit (catalog no. VPB-1002; Lonza).

For visualization of early endosomes in live cells, cells were transiently transduced with the RFP-Rab5 expression vector (CellLight ${ }^{\mathrm{TM}}$ Early Endosomes-RFP fusion construct (catalog no. C10587; Invitrogen/Thermo Fisher Scientific) according to the manufacturer's instructions. In brief, cells were plated at a density of $1 \times 10^{4}$ cells in $35-\mathrm{mm}$ glassbottomed dishes (catalog no. P 35G-0-14-C; MatTek Co., Ashland, MA, USA) 1 day before transduction. The cells were incubated in EGM-2 medium containing $5 \mu$ of BacMam reagent at $37{ }^{\circ} \mathrm{C}$ under $5 \% \mathrm{CO}_{2}$ for $12-16 \mathrm{~h}$, following which the medium was replaced with fresh EGM-2. The experiment was carried out after $4 \mathrm{~h}$ of recovery.

\section{Fluorescein isothiocyanate-labeled dextran uptake assay}

Human umbilical vein endothelial cells that had been grown in EGM-2 overnight after plating were incubated with 0.2 or $0.5 \mathrm{mg} / \mathrm{ml}$ fluorescein isothiocyanate (FITC)-dextran (MW 70 kDa; catalog no.\# 60842-46-8; Sigma, St. Louis, MO, USA) or Alexa Fluor 647-dextran (MW 10 kDa; catalog no. D 22914; Molecular Probes/Thermo Fisher Scientific, Eugene, OR, USA ) at $37{ }^{\circ} \mathrm{C}$ for the specified time period. After washing with $\mathrm{Ca}^{2+}$ - and $\mathrm{Mg}^{2+}$-free Dulbecco's phosphate buffered saline (PBS), the cells were fixed with $4 \%$ paraformaldehyde (PFA; FUJIFILM Wako Pure Chemical Corp., Tokyo, Japan) and stained with 4',6-diamidino2-phenylindole (DAPI) (catalog no. D1306; Molecular Probes). For the pulse-chase analysis of dextran, cells were incubated for $1 \mathrm{~h}$ with $0.5 \mathrm{mg} / \mathrm{ml}$ FITC-dextran at $37{ }^{\circ} \mathrm{C}$ in growth medium, washed twice with fresh growth medium and placed in a $\mathrm{CO}_{2}$ incubator. At the indicated time points, cells were fixed with $4 \%$ PFA for 10 min and stained with DAPI. Fluorescence images were captured with confocal microscopy as described in section Confocal microscopy and super-resolution radial fluctuation-Stream imaging. Image acquisition and processing were performed with iQ software (Andor Technology Ltd., Belfast, Northern Ireland).
FITC-dextran uptake was quantified by using the 'Analyze Particles' feature in Fiji (Image J) software.

\section{Western blotting}

Total cell lysates were prepared by scraping PBS-washed cells in $2 \times$ Laemmli sodium dodecyl sulfate-polyacrylamide gel electrophoresis (SDS-PAGE) sample buffer on ice $48 \mathrm{~h}$ after siRNA transfection. The samples were separated in $8 \%$ SDS-PAGE gels, followed by electrotransfer onto PVDF membranes (catalog no. IPV00010; Immobilon-P, Millipore-Merck, Nottingham, UK) using the Trans-Blot Turbo blotting system (Bio-Rad, Hercules, CA, USA). The membranes were blocked in $5 \%$ bovine serum albumin and $0.1 \%$ Tween- 80 in Tris-buffered saline for $1 \mathrm{~h}$ and incubated with specific antibodies at $4{ }^{\circ} \mathrm{C}$ overnight. The antibodies used were PI3K-C2 $\alpha$ (1:1000) (catalog no.12402; Cell Signaling Technology [CST], Danvers, MA, USA.), PI3K-C2 $\beta$ (1:1000) (catalog no. 611342; BD Transduction Laboratories, BD Biosciences, San Diego, CA USA), p110 $\alpha$ (1:1000) (catalog no. 4249; CST), Vps34 (1:1000) (catalog no. 4263; CST), clathrin heavy chain (1:1000) (catalog no. ab21679; Abcam) and glyceraldehyde 3-phosphate dehydrogenase (1:1000) (catalog no. 016-25523; FUIJIFILM-Wako Pure Chemical Corp.). The membranes were incubated with alkaline phosphatase-conjugated secondary antibodies anti-rabbit immunoglobulin (Ig) G antibody (1:1000) (catalog no. 7054; CST) and anti-mouse IgG antibody (1:1000) (catalog no. 7056; CST) for $1 \mathrm{~h}$ at room temperature, and the protein bands were visualized by a color reaction using a nitro-blue tetrazolium/5-bromo-4-choloro-3'-indolylphosphate p-toluidine (both FUJIFILM Wako Pure Chemical Corp.) system. Protein band intensities were determined using Image Studio lite software (LI-COR Biosciences, Lincoln, NB, USA).

\section{Immunostaining}

Human umbilical vein endothelial cells were plated onto type-I collagen-coated glass-bottomed dishes and allowed to adhere to dishes in EGM-2 overnight. The cells were then rinsed with PBS once and fixed with $4 \%$ PFA in $0.1 \mathrm{M}$ phosphate buffer ( $\mathrm{pH}$ 7.4) for $10 \mathrm{~min}$ at room temperature, followed by permeabilization with $0.3 \%$ TritonX-100 in PBS for 15 min or in $90 \%$ chilled methanol for 5 min. After blocking in 5\% normal goat serum (FUJIFILM Wako Pure Chemical Corp.)/0.3\% TritonX-100 in PBS, the cells were incubated with the indicated primary antibodies overnight at $4{ }^{\circ} \mathrm{C}$. The antibodies used were PI3K-C2 $\beta$ (1:500) (catalog no. 611342; BD Transduction Laboratories), clathrin heavy chain (1:400) (catalog no. ab21679; Abcam), clathrin heavy chain (1:400) (catalog no.MA1-065; Thermo Fisher Scientific), EEA1 (1:200) (catalog no. 610456; BD BioSciences), EEA1 (1:200) (catalog no. PA1-063A; Thermo Fisher 


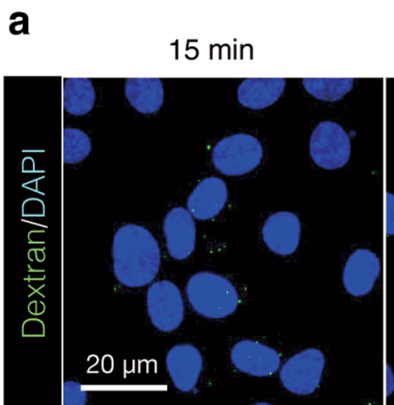

$30 \mathrm{~min}$

$45 \min$

$60 \min$

$120 \mathrm{~min}$


b

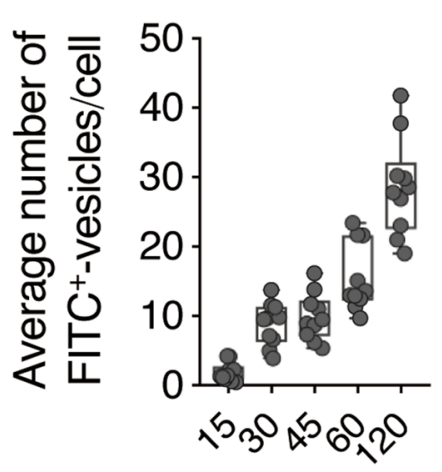

Incubation time (min)
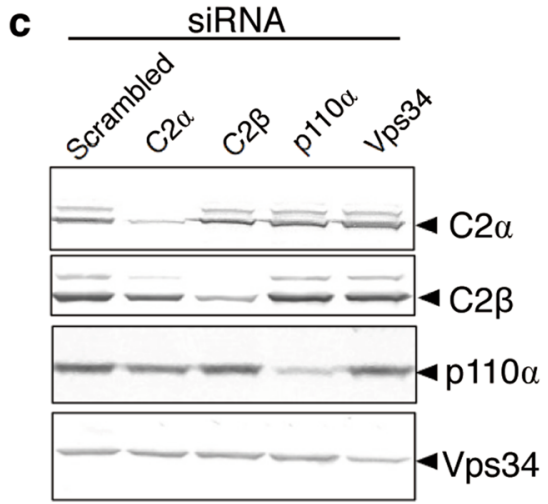



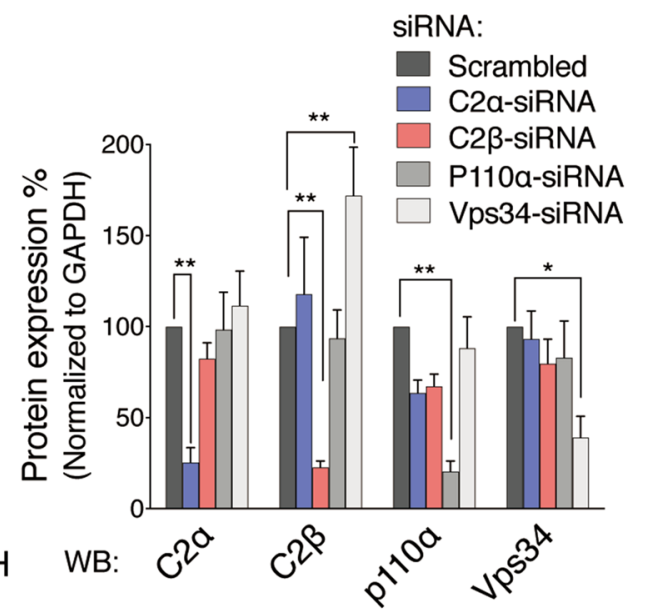

d SiRNA

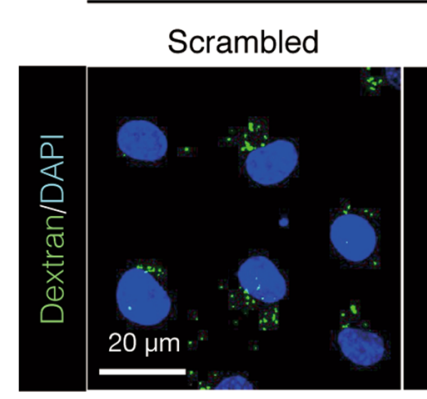

$\mathrm{C} 2 \beta$

$\mathrm{p} 110 \alpha$

Vps34



f

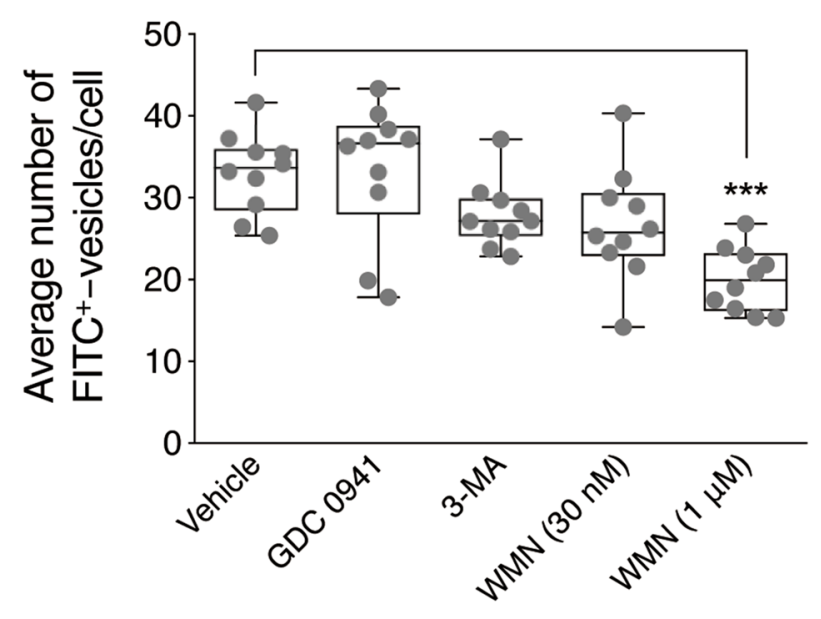


४Fig. 1 Class II phosphoinositide 3-kinase (PI3K)-C2 $\alpha$ and PI3K-C2 $\beta$ are necessary for fluid-phase pinocytosis in human umbilical vein endothelial cells (HUVECs). a, b Time-dependent uptake of fluorescein isothiocyanate (FITC)-dextran. Cells were incubated with FITCdextran $(0.2 \mathrm{mg} / \mathrm{ml})$ in growth medium at $37{ }^{\circ} \mathrm{C}$ for the indicated time periods, followed by fixation with $4 \%$ paraformaldehyde. Nuclei were stained with 4',6-diamidino-2-phenylindole (DAPI; blue). Scale bar: $20 \mu \mathrm{m}$. a Representative images showing internalized FITCdextran (green dots) at each time point. b Quantified data $(n=50-60$ cells were analyzed in each group). The green dots were quantified as described in the Materials and methods. c Small interfering RNA (siRNA)-mediated knockdown of PI3K isoforms. Representative western blots $(W B)$ showing siRNA-mediated knockdown of PI3K protein expression after $48 \mathrm{~h}$ post-transfection (left). Cells were transfected with either PI3K-C $2 \alpha-$, PI3K-C2 $\beta$-, p1 $10 \alpha$ - or Vps34-specific siRNA or scrambled siRNA. Glyceraldehyde 3-phosphate dehydrogenase $(G A P D H)$ was used as loading control. Quantified data of protein expression levels (right). d, e Effects of knockdown of different PI3K isoforms on FITC-dextran uptake. Cells were incubated with FITC-dextran for $45 \mathrm{~min}$. d representative images, e quantified data. f Effects of different PI3K inhibitors on FITC-dextran uptake. Cells were pretreated with the inhibitors GDC-0941 $(0.1 \mu \mathrm{M})$, 3-methyladenine $(3-M A ; 1 \mathrm{mM})$ or wortmannin $(W M N ; 30 \mathrm{nM}$ and $1 \mu \mathrm{M})$ for $30 \mathrm{~min}$ and then subjected to the FITC-dextran uptake assay. Scale bar: $20 \mu \mathrm{m}$. Data are presented as the mean \pm standard error of the mean (SEM) in $\mathbf{c}$ and as the median and interquartile range (IQR) in $\mathbf{b}, \mathbf{e}$ and $\mathbf{f}$. Asterisks indicate statistical significance between the indicated groups: $* p<0.05, * * p<0.01, * * * p<0.001$

Scientific), Rab7 (1:100) (catalog no. 9367; CST), LAMP1 (1:200) (catalog no. 328601; BioLegend, San Diego, CA, USA), LC3B (1:400) (catalog no. 3868; CST) and ITSN1 (1:400) (catalog no. ab118262; Abcam). After washing with $0.1 \%$ TritonX-100 in PBS, the cells were incubated with appropriate Alexa Fluor-conjugated secondary antibodies (Molecular Probes) for $1 \mathrm{~h}$ at room temperature. For F-actin staining, HUVECs were washed twice with prewarmed PBS, fixed with $4 \%$ PFA and permeabilized in $0.2 \%$ Triton X-100 for $5 \mathrm{~min}$. The cells were then stained with iFluor 488-conjugated phalloidin (catalog no. 0549; Cayman Chemical, Ann Arbor, MI, USA) or Alexa Fluor 594-conjugated phalloidin (catalog no. 12381; Molecular Probes) according to the manufacturer's instruction. The cells were counterstained with DAPI for $30 \mathrm{~min}$.

\section{Confocal microscopy and super-resolution radial fluctuation-Stream imaging}

Results from Figs. 1, 2 and 5 were carried out on a custom confocal microscope (modified inverted IX70 microscope; Olympus Corp., Tokyo, Japan) equipped with an UPLSAPO 60X/NA1.35-oil objective, a confocal disk-scanning unit (CSU10; Yokogawa, Tokyo, Japan) and an EMCCD camera (iXon DV887; Andor Technology Ltd.) as described previously [13]. The acquisition and process were controlled by iQ software (Andor Technology Ltd.). All other observations and live-cell imaging were carried out on an inverted Nikon Eclipse Ti2 confocal microscope (Nikon Instruments/
Nikon Corp., Tokyo, Japan) equipped with the Perfect Focus System, attached to an Andor Dragonfly spinning-disk unit, Andor EMCCD camera (iXon DU888; Andor Technology Ltd.) and a laser unit (Coherent Inc., Santa Clara, CA, USA). An oil-immersion objective (PlanApo 60X; NA 1.4; Nikon Corp.) was used for all experiments. Excitation for BFP/DAPI, GFP/mEmerald/Alexa 488, mRFP/mCherry/ Alexa 568 and Alexa 647 chromophores was provided by a 405-, 488-, 561- and 637-nm laser, respectively. Superresolution imaging of fixed cells was performed using an a Dragonfly confocal microscope in super-resolution radial fluctuation (SRRF)-Stream mode (Andor Technology Ltd.). Live-cell imaging of multicolor-labeled cells plated on collagen-coated glass-bottom dishes was performed in EGM-2 containing $2 \% \mathrm{FBS}$ at $37{ }^{\circ} \mathrm{C}$ with $5 \% \mathrm{CO}_{2}$ in a humidified incubator (Tokai-Hit Co. Ltd., Shizuoka, Japan) using the confocal microscope in SRRF-Stream mode as described above. Live-cells were maintained in the imaging incubator for up to $30 \mathrm{~min}$. Time-lapse images were taken at 10 -s intervals using Andor Fusion software, and movies were prepared at a frequency of 20 flames/s using Imaris software (Bitplane; Oxford Instruments, Abingdon, UK). To minimize sample drift in the $z$-direction over time, we applied a motorized Piezo stage controlled by a near infrared-light adjusted using the Perfect Focus System (Nikon Corp.). Images were quantified using Image J software, and the area and shape characteristics were measured using the Analyze Particles in Fiji (ImageJ) software.

\section{Statistical analysis}

Statistical analysis was performed using Prism 7 software (GraphPad Software, San Diego, CA, USA). Three or more sample numbers were acquired for the quantitative western blot analysis and image analysis. Data were presented as the mean \pm standard error of the mean or as the median and interquartile range with error bars denoting minimal and maximal values. For comparison between multiple groups, one- or two-way analysis of variance followed by the Bonferroni post hoc test was used unless stated otherwise. A $p$ value of $<0.05$ was considered to be statistically significant.

\section{Results}

\section{Class II PI3K-C2 $\alpha$ and PI3K-C2 $\beta$ are necessary for pinocytosis}

We studied the roles of PI3K-C $2 \alpha$ and $-\mathrm{C} 2 \beta$ in pinocytic activity at steady-state in HUVECs, a well-characterized human primary vascular endothelial cell model. Pinocytic activity was evaluated by determining the time-dependent uptake of a fluid-phase marker, FITC-dextran, into cells. 

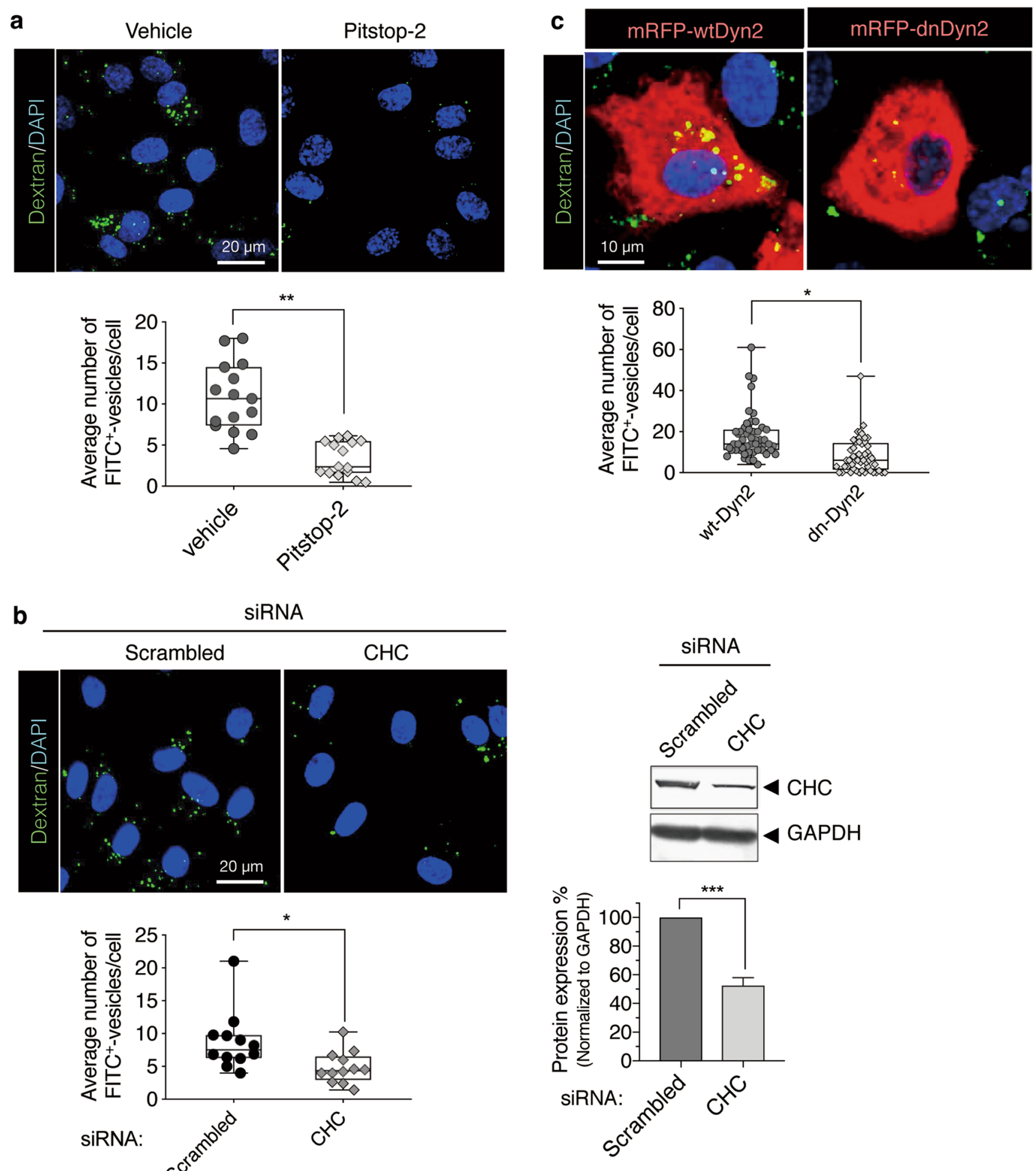

FITC-dextran uptake was observed to increase in a timedependent manner (Fig. 1a, b). To study the roles of PI3K isoforms in pinocytosis, we investigated the effects of siRNA-mediated knockdown of individual PI3K isoforms on FITC-dextran uptake. The siRNAs specific to class II

PI3K-C $2 \alpha$ and $-\mathrm{C} 2 \beta$, class I p110 $\alpha$, and class III Vps34 effectively and specifically inhibited the protein expression of the respective PI3K isoform (Fig. 1c). Knockdown of PI3K-C $2 \alpha$ or PI3K-C $2 \beta$ partially but significantly (53 and $70 \%$, respectively) decreased FITC-dextran uptake, 
4Fig. 2 Pinocytosis is partially dependent on clathrin and dynamin in HUVECs. a Effects of the clathrin inhibitor Pitstop-2 on FITCdextran uptake. Cells were pretreated with either Pitstop-2 $(20 \mu \mathrm{M})$ or vehicle for $30 \mathrm{~min}$ and then subjected to the FITC-dextran uptake assay. Upper, Representative images (scale bar: $20 \mu \mathrm{m}$ ); lower, quantified data. b Effects of clathrin heavy chain $(\mathrm{CHC})$ knockdown on FITC-dextran uptake. Cells were transfected with either scrambled or CHC-siRNA. Left upper, Representative images; left lower, quantified data of FITC-dextran uptake; right upper, representative blots of $\mathrm{CHC}$ protein; right lower, quantified data of protein expression levels. $\mathbf{c}$ Effects of the expression of monomeric red fluorescent protein (mRFP)-tagged dominant negative dynamin-2 (K44A) mutant $(d n-D y n 2)$ on FITC-dextran uptake. Cells were transfected with either mRFP-tagged wild type dynamin2 (wt-Dyn2) or dn-Dyn2, and $24 \mathrm{~h}$ later subjected to FITC-dextran uptake. Upper, Representative images of cells. Nuclei were stained with DAPI (blue). Lower, Quantified data of FITC-dextran uptake. $n=50-60$ cells were analyzed in each group. Data are presented as the median and IQR in $\mathbf{a}, \mathbf{b}$, lower left and $\mathbf{c}$, and as the mean \pm SEM in $\mathbf{b}$, lower right. Asterisks indicate statistical significance between the indicated groups: ${ }^{*} p<0.05$, $* * p<0.01, * * * p<0.001$

compared with scrambled siRNA transfection (Fig. 1d, e). In contrast, knockdown of either p110 $\alpha$ or Vps 34 did not inhibit FITC-dextran uptake. We also studied the effects of pharmacological PI3K inhibitors on pinocytic activity. The treatment of cells with a higher dose $(1 \mu \mathrm{M})$ of the panPI3K inhibitor WMN caused a reduction in FITC-dextran uptake compared with cells treated with vehicle. However, a lower dose $(30 \mathrm{nM})$ of WMN, the class I PI3K inhibitor GDC-0941 or the Vps34-specific inhibitor 3-MA failed to inhibit FITC-dextran uptake (Fig. 1f). These data suggest that class II PI3K-C $2 \alpha$ and PI3K-C $2 \beta$, but not class I p1 $10 \alpha$ or class III Vps34, are involved in fluid-phase pinocytosis in HUVECs.

\section{Pinocytosis in HUVECs is partially dependent on clathrin and dynamin}

We examined whether pinocytosis at steady-state in HUVECs required clathrin and dynamin or not. The clathrin inhibitor, Pitstop-2, inhibited FITC-dextran uptake by approximately $69 \%$ (Fig. 2a). Similarly, siRNA-mediated knockdown of the CHC inhibited FITC-dextran uptake by approximately 55\% (Fig. 2b). Furthermore, the expression of the dominant negative mutant (K44A) of dynamin-2 (dn-Dyn2) inhibited the FITC-dextran uptake by approximately 53\% (Fig. 2c). These observations indicate that pinocytosis in HUVECs is at least partially mediated by clathrin- and dynamin-dependent processes. Interestingly, the extent of inhibition by the inhibitor, of the knockdown and of the mutant protein were roughly similar to those induced by knockdown of PI3K-C $2 \alpha$ and PI3K-C2 $\beta$ (Fig. 1e).

\section{Partially overlapping subcellular localization of PI3K-C2 $\alpha$ and PI3K-C2 $\beta$}

The subcellular localization of PI3K-C $2 \alpha$ and PI3K-C $2 \beta$ in HUVECs that expressed GFP-C $2 \alpha$ and mCherry-C $2 \beta$ was studied using super-resolution microscopy. GFP-C $2 \alpha$ was distributed as fine puncta widely throughout the cells, whereas mCherry-C2 $\beta$ was enriched mainly in the perinuclear region as coarse puncta, in the peripheral regions surrounding podosomes as coarse and fine puncta and in the plasma membrane (Fig. 3a). A substantial portion of mCherry-C2 $\beta$ in the cell periphery was closely associated with F-actin structures (membrane ruffles and small F-actin assembly [actin patches]) (Fig. 3b, right). In contrast, GFP-C $2 \alpha$ was barely associated with the F-actin structures (Fig. 3b, left). The punctate fluorescence of GFP-C $2 \alpha$ overlapped strikingly with clathrin-coated pits and vesicles. A part of the GFP-C2 $\alpha$ fluorescence was co-localized with EEA1-positive $\left(\mathrm{EEA1}^{+}\right.$) early endosomes (EE), and to much lesser extents with $\mathrm{Rab}^{+}$late endosomes (LE), LAMP1 ${ }^{+}$ lysosomes (LY), and $\mathrm{LC} \mathrm{B}^{+}$autophagosomes (AP) (Fig. 3c). Our research group and others have previously demonstrated the co-localization of PI3K-C $2 \alpha$ with clathrin-coated structures and EE, using anti-PI3K-C $2 \alpha$ immunostaining [13, 21-23]. In contrast, PI3K-C2 $\beta$ signals were less frequently co-localized with clathrin-coated pits and vesicles compared with GFP-C $2 \alpha$ and slightly co-localized with EE, LE and LY. However, perinuclear PI3K-C $2 \beta$ was highly co-localized with LC3B ${ }^{+}$-AP (Fig. 3d). Thus, GFP-C $2 \alpha$ and mCherry$\mathrm{C} 2 \beta$ showed significantly different subcellular localization, suggesting that PI3K-C $2 \alpha$ and $-\mathrm{C} 2 \beta$ may differentially control pinocytic processes. We also determined the subcellular localization of endogenous PI3K-C2 $\beta$ using anti-PI3K-C2 $\beta$ immunostaining. Endogenous PI3K-C $2 \beta$ was partially colocalized with F-actin structures, clathrin-coated structures and $\mathrm{LC} 3 \mathrm{~B}^{+} \mathrm{AP}$ and less frequently with EEA $1^{+} \mathrm{EE}$ (Fig. 4), which is similar to the results obtained with mCherry-C2 $\beta$ expression (Fig. 3d).

\section{$\mathrm{PI} 3 \mathrm{~K}-\mathrm{C} 2 \alpha$ and $-\mathrm{C} 2 \beta$ are required for clathrin-mediated pinocytosis}

To gain further insight into the regulation of pinocytosis by PI3K-C $2 \alpha$ and PI3K-C $2 \beta$, we examined the effects of double knockdown of PI3K-C $2 \alpha$ and PI3K-C $2 \beta$ mRNAs on pinocytosis. The double knockdown of PI3K-C $2 \alpha$ and $-\mathrm{C} 2 \beta$ gave similar extents of suppression of $\mathrm{PI} 3 \mathrm{~K}-\mathrm{C} 2 \alpha$ and $-\mathrm{C} 2 \beta$ protein expression and the FITC-dextran uptake as with the single knockdown of either PI3K-C $2 \alpha$ or PI3K-C2 $\beta$ (Fig. 5a). The next step was to examine which of clathrin-dependent and -independent endocytic mechanisms in pinocytosis requires 

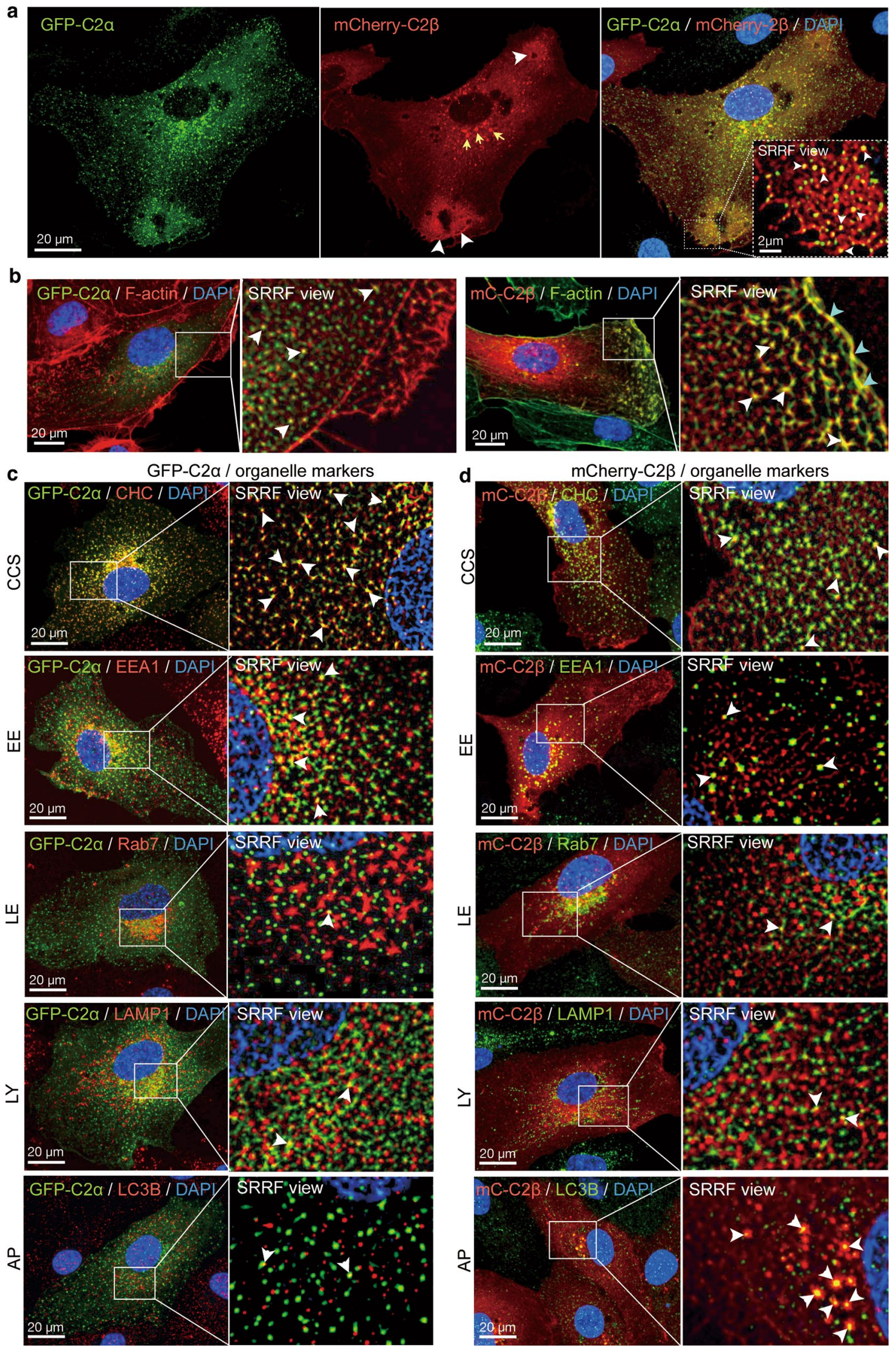
4Fig. 3 Partially overlapping subcellular localization of PI3K-C2 $\alpha$ and PI3K-C2 $\beta$ in HUVECs. a Cells were cotransfected with the expression vectors of green fluorescent protein $(G F P)-\mathrm{C} 2 \alpha$ and mCherry$\mathrm{C} 2 \beta$ and imaged by confocal microscopy coupled with super-resolution radial fluctuation (SRRF)-Stream mode. Nuclei were stained with DAPI (blue). Note the cytoplasmic fine puncta of GFP-C $2 \alpha$ (left) and coarse and fine puncta of mCherry-C2 $\beta$ (middle) surrounding the podosomes and on the plasma membrane (white arrowheads) and perinuclear coarse puncta of mCherry-C2 $\beta$ (yellow arrows). White arrowheads in the SRRF view of the merged image indicate the co-localization of GFP-C $2 \alpha$ and mCherry-C $2 \beta$. b Representative confocal microscopic images of GFP-C $2 \alpha$ or mCherry-C2 $\beta$ and F-actin. Cells transfected with the expression vectors of GFP-C $2 \alpha$ and mCherry-C2 $\beta$ were stained with iFluor 488-conjugated phalloidin (green) or Alexa Fluor 594-conjugated phalloidin (red). Nuclei were stained with DAPI. White arrowheads indicate the co-localization of F-actin and GFP-C $2 \alpha$ or mCherry-C2 $\beta$. Blue arrowheads indicate the co-localization of F-actin and mCherry-C2 $\beta$ in the membrane ruffle. c, $\mathbf{d}$ Co-localization of GFP-C $2 \alpha$ (c) or mCherry-C2 $\beta$ (d) with organelle markers. Cells expressing either GFP-C $2 \alpha$ or mCherry-C2 $\beta$ were stained with antibodies against $\mathrm{CHC}$ (clathrin-coated structures $[C C S]$ ), EEA1 (early endosomes $[E E]$ ), Rab7 (late endosomes $[L E]$ ), LAMP1 (lysosomes $[L Y]$ ) and LC3B (autophagosomes [AP]). Nuclei were stained with DAPI (blue). White arrowheads in SRRF views indicate the co-localization of GFP-C $2 \alpha$ or mCherry-C $2 \beta$ with each organelle marker

PI3K-C2 $\alpha$ and -C2 $\beta$. Knockdown of CHC inhibited FITCdextran uptake. Double knockdown of $\mathrm{CHC}$ and either of $\mathrm{PI} 3 \mathrm{~K}-\mathrm{C} 2 \alpha$ and PI3K-C2 $\beta$ did not result in further inhibition of FITC-dextran uptake compared with inhibition by $\mathrm{CHC}$ single knockdown (Fig. 5b). These findings suggest that both PI3K-C $2 \alpha$ and -C $2 \beta$ are required for clathrin-dependent, but not clathrin-independent, pinocytosis. Since single knockdown of either PI3K-C $2 \alpha$ or $-\mathrm{C} 2 \beta$ resulted in complete inhibition of the clathrin-mediated pinocytosis, we concluded that the two isoforms play non-redundant, distinct roles in clathrin-mediated pinocytosis.

Live cell super-resolution imaging of the cells transfected with either GFP-C $2 \alpha$ or GFP-C $2 \beta$ and mRFP-CLC showed that GFP-C $2 \alpha$ formed dynamic puncta that were mostly co-localized with mRFP-CLC (Fig. 5c and Electronic Supplementary Material [ESM] movie 1 with movie legends (Supplementary material 1$)$ ). GFP-C $2 \beta$ was also colocalized with mRFP-CLC puncta, but the co-localization was less abundant than in the case of PI3K-C $2 \alpha$ (Fig. $5 \mathrm{~d}$ and ESM movie 2 with movie legends). The serial, magnified images showed that PI3K-C $2 \alpha$ and $-\mathrm{C} 2 \beta$ were recruited to mRFP-CLC puncta and then the clathrin was disassembled, followed by the dissociation of GFP-C $2 \alpha$ and GFP-C $2 \beta$ (Fig. 5c, d, lower panels, and e). The fluid-phase marker Alexa 647-dextran was found in vesicles that were double-positive for mRFP-CLC and either GFP-C $2 \alpha$ or $-\mathrm{C} 2 \beta$ (Fig. 5f), thereby providing morphological evidence that the dextran was pinocytosed, at least in part, into PI3K-C $2 \alpha$ and -C2 $\beta$-associated clathrin-coated vesicles.


Fig. 4 Immunofluorescent staining of endogenous PI3K-C2 $\beta$ in HUVECs. Cells were double-immunostained with anti-PI3K-C2 $\beta$ (green) and Alexa Flour 594-conjugated phalloidin (red), anti-CHC (red), anti-EEA1 (red) or anti-LC3B (red), and imaged by confocal microscopy coupled with SRRF-Stream mode. Nuclei were stained with DAPI (blue). Scale bar: $20 \mu \mathrm{m}$. White arrowheads in SRRF views indicate the co-localization of endogenous PI3K-C2 $\beta$ with each organelle marker

Since clathrin-coated pinocytic vesicles are transported to EE, we examined the effects of knockdown of PI3K$\mathrm{C} 2 \alpha$ and $-\mathrm{C} 2 \beta$ on FITC-dextran accumulation in EE in the cells expressing the EE marker mRFP-Rab5. Knockdown 
a

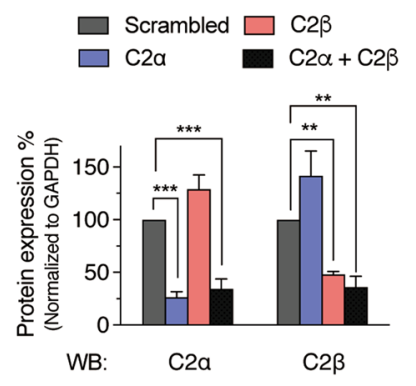

C

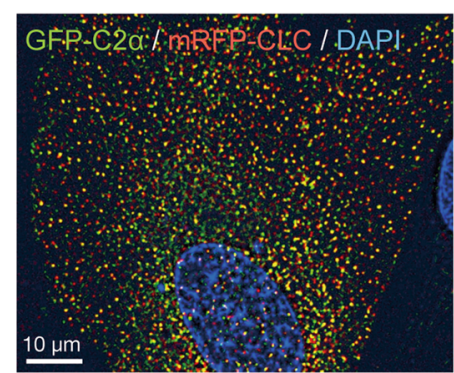

Clathrin

$\mathrm{C} 2 \mathrm{a}$

Merged

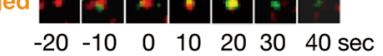





Clathrin

$\mathrm{C} 2 \beta$

Merged $\%$

b




- Clathrin - C2 $\beta$

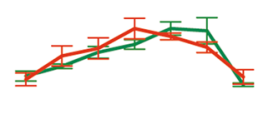

$-30-20-100 \quad 102030$ Time (sec)
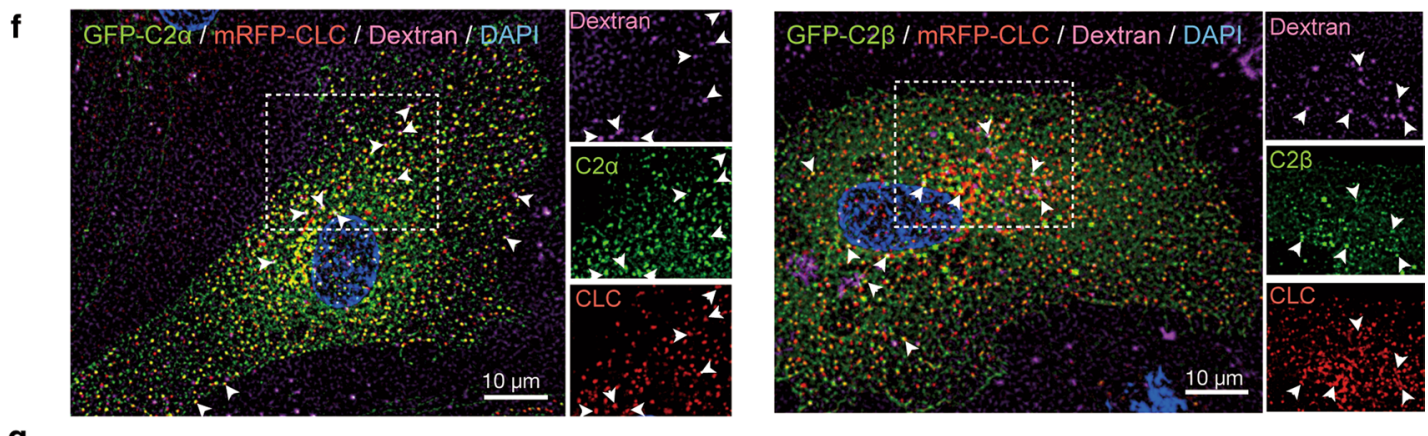

g
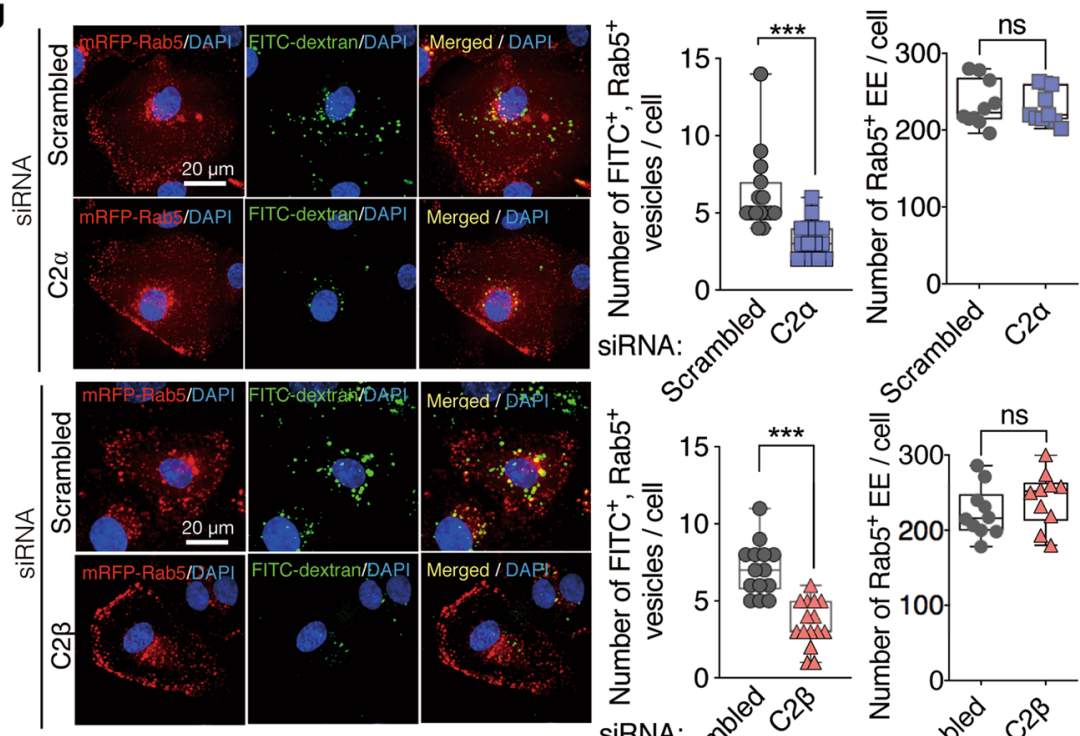

siRnA:
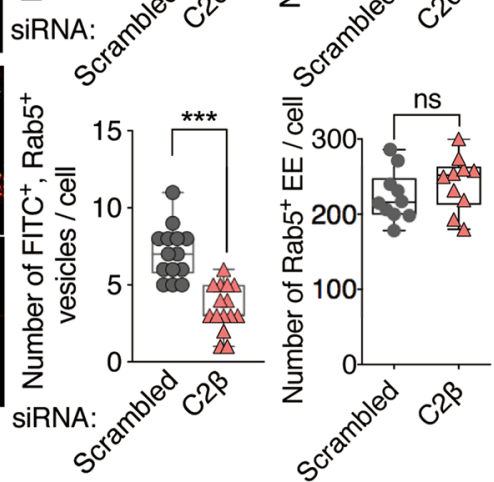

h




4Fig. 5 PI3K-C2 $\alpha$ and PI3K-C2 $\beta$ are required for clathrin-mediated pinocytosis. a Effects of double knockdown of PI3K-C2 $\alpha$ and -C2 $\beta$ on FITC-dextran uptake. Cells were transfected with scrambled, PI3K-C $2 \alpha$ or $-\mathrm{C} 2 \beta$ siRNAs (50 $\mathrm{nM}$ each) or with the combination of $\mathrm{C} 2 \alpha$ - and $\mathrm{C} 2 \beta$-siRNAs ( $25 \mathrm{nM}$ each). Left, Relative protein expression levels of PI3K-C2 $\alpha$ and $-\mathrm{C} 2 \beta$ determined by western blotting. Right, FITC-dextran uptake, b No effect of $\mathrm{C} 2 \alpha-$ and $\mathrm{C} 2 \beta$ knockdown in CHC-depleted HUVECs. The single and double knockdown was performed as described in the Materials and Methods. After $48 \mathrm{~h}$, cells were subjected to the FITC-dextran uptake assay as described in a. c, $\mathbf{d}$ Super-resolution microscopic images of cells transfected with the expression vectors of mRFP-clathrin light chain (mRFP-CLC, red) and either GFP-C $2 \alpha$ (green in c) or GFP-C $2 \beta$ (green in d). Nuclei were stained with DAPI (blue). Scale bar: $10 \mu \mathrm{m}$. Lower, Representative serial images showing the dynamics of PI3K$\mathrm{C} 2 \alpha(\mathbf{c})$ and $-\mathrm{C} 2 \beta(\mathbf{d})$ association with clathrin. Time zero was set as the peak of clathrin recruitment. See also Electronic Supplementary Material movies 1 and 2 with movie legends. e Quantified data of the lower images in $\mathbf{c}$ and $\mathbf{d}$. $n=3$ and 4 events for $\mathrm{C} 2 \alpha /$ clathrin and $\mathrm{C} 2 \beta /$ clathrin, respectively. f Co-localization of GFP-C2 $\alpha$ (left) or -C2 $\beta$ (right) in clathrin-coated pinocytic vesicles. Super-resolution microscopic images of cells expressing GFP-C $2 \alpha$ (left) or -C2 $\beta$ (right) and mRFP-CLC that were subjected to Alexa Fluor 647-dextran uptake. White arrowheads indicate dextran-containing $\mathrm{C} 2 \alpha$ - or C2 $\beta$-positive pinosomes. $\mathrm{g}$ Effects of either PI3K-C $2 \alpha$ or $-\mathrm{C} 2 \beta$ knockdown on FITC-dextran accumulation in EE. Cells were transfected with scrambled, PI3K-C $2 \alpha$ or $-\mathrm{C} 2 \beta$ siRNAs. 24 h later, the knocked down cells were infected with RFP-Rab5 baculoviral vector, followed by FITCdextran uptake assay. Left, representative images and right, quantified data of FITC-dextran ${ }^{+}$, mRFP-Rab5 ${ }^{+}$vesicles and mRFP-Rab5 ${ }^{+}$ vesicles. $\mathbf{h}$ Time-dependent degradation of uptaked FITC-dextran in PI3K-C $2 \alpha-$ and $-\mathrm{C} 2 \beta$-depleted cells. Cells that had been transfected with indicated siRNAs were subjected to FITC-dextran pulse-chase experiments as described in the Materials and methods. Nuclei were stained with DAPI (blue). Data are presented as the median and IQR in a, right, $\mathbf{b}$ and $\mathbf{g}$ and as the mean \pm SEM in $\mathbf{a}$, left, $\mathbf{e}$ and $\mathbf{h}$. Asterisks indicate statistically significant difference between the indicated groups: ${ }^{*} p<0.05,{ }^{* *} p<0.01, * * * p<0.001 ; n s$ not significant

of either PI3K-C $2 \alpha$ or PI3K-C2 $\beta$ reduced the numbers of FITC-dextran puncta in Rab5 ${ }^{+}$EE after 60 min of FITCdextran uptake without any change in the number of Rab5 ${ }^{+}$ EE (Fig. 5g). We determined the time course of degradation of internalized FITC-dextran using pulse-chase experiments. After 60 min of FITC-dextran uptake, the cells were washed and monitored for up to $8 \mathrm{~h}$ for the FITCdextran remaining in the cells. The intensity of FITC-dextran within either PI3K-C2 $\alpha$ - or PI3K-C $2 \beta$-depleted cells gradually decreased with a similar time course, compared with scrambled siRNA-transfected cells (Fig. 5h), suggesting that degradation of the uptaked FITC-dextran was not altered by knockdown of PI3K-C $2 \alpha$ or -C2 $\beta$. These findings collectively suggest that both PI $3 \mathrm{~K}-\mathrm{C} 2 \alpha$ and PI $3 \mathrm{~K}-\mathrm{C} 2 \beta$ are involved in the early step(s) of the clathrin-mediated pinocytic pathway, namely the process up to FITC-dextran delivery to the Rab5 ${ }^{+}$-EE.
$\mathrm{PI} 3 \mathrm{~K}-\mathrm{C} 2 \beta$ is required for the formation of pinocytic vesicle-associated actin filaments

Based on our results showing the co-localization of class II PI3Ks, particularly PI3K-C2 $\beta$, with F-actin (Fig. 3) and on previous reports noting the requirement of actin polymerization at clathrin-coated pits and vesicles for pit maturation and vesicle formation [24-26], we studied the roles of $\mathrm{PI} 3 \mathrm{~K}-\mathrm{C} 2 \alpha$ and $-\mathrm{C} 2 \beta$ in F-actin formation at clathrin-coated pits, using super-resolution microscopy. The cells that had been transfected with mRFP-CLC and the GFP-tagged F-actin-binding peptide mEmerald-Lifeact were subjected to uptake of Alexa 647-dextran. As in the case of FITCdextran uptake, knockdown of PI3K-C $2 \alpha$ or $-\mathrm{C} 2 \beta$ substantially inhibited Alexa 647-dextran uptake (Fig. 6a-d). Many of the dextran-containing pinocytic vesicles were associated with F-actin (actin patches) (Fig. 6a). A substantial portion of clathrin-coated pits and vesicles was associated with actin patches. Knockdown of PI3K-C $2 \beta$ greatly reduced the formation of clathrin-associated actin patches (Fig. 6a-c, e). Moreover, knockdown of PI3K-C2 $\beta$, but not of PI3K-C $2 \alpha$, reduced the co-localization of actin patches and dextran (Fig. 6a-c, f). Consistent with this, there were relatively fewer dextran-positive pinocytic vesicles in PI3KC2 $\beta$-depleted cells that were associated with F-actin (white arrowheads in Fig. 6a, c). Instead, there were relatively more pinocytic vesicles in PI3K-C2 $\beta$-depleted cells that were not associated with F-actin (yellow arrows). These observations suggest that PI3K-C2 $\beta$ facilitated actin patch formation at clathrin-coated structures to promote clathrin-mediated pinocytosis.

\section{Overexpression of PI3K-C2 $\beta$ but not PI3K-C2 $\alpha$ enhances the uptake of dextran}

We studied whether overexpression of mCherry-PI3K-C2 $\alpha$ and $-\mathrm{C} 2 \beta$ affected pinocytosis. Intersectin-1 (ITSN1), which was originally found to be localized in clathrin-coated pits [27], has been very recently identified as a multifunctional scaffold protein that is required for actin patch formation [28]. The ITSN1 has also been shown previously to bind PI3K-C2 $\beta$ [29]. Therefore, we compared the effects of PI3K$\mathrm{C} 2 \alpha$ and $-\mathrm{C} 2 \beta$ overexpression with and without expression of GFP-tagged ITSN1 (GFP-ITSN1). mCherry-C $2 \beta$ expression increased dextran uptake only when GFP-ITSN1 was co-expressed (Fig. 7a, b). Interestingly, the expression of GFP-ITSN1 recruited mCherry-C2 $\beta$ from the cytoplasmic pool to GFP-ITSN1-localized structures (Fig. 7a). In contrast, PI3K-C $2 \alpha$ overexpression did not increase dextran uptake or induce PI3K-C $2 \alpha$ recruitment to dot-like structures 



d

Dextran uptake

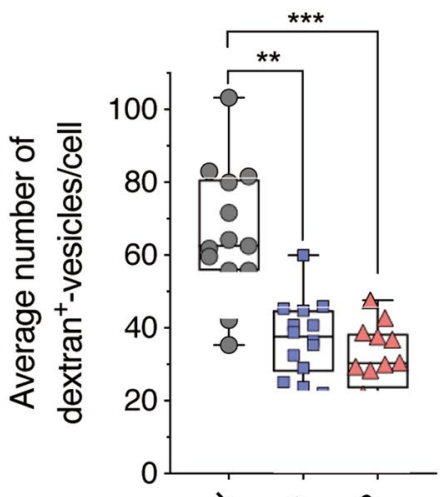

siRNA:

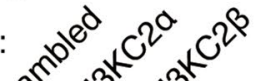


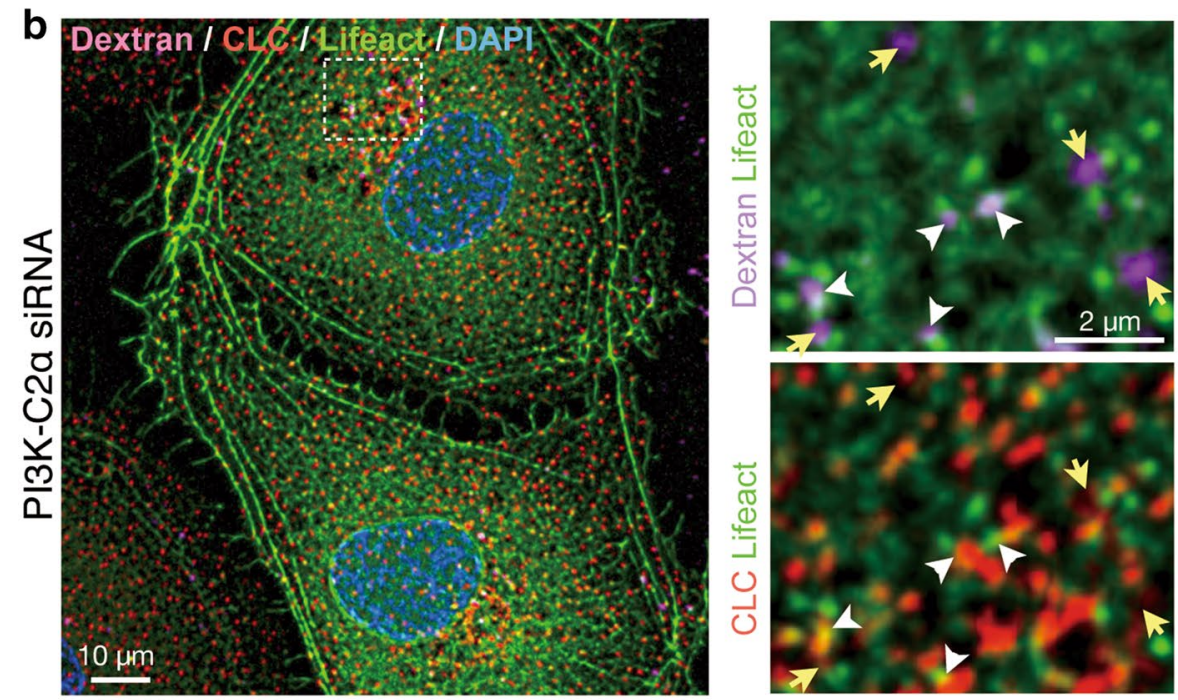

e

Lifeact/ CLC

(Actin patches)
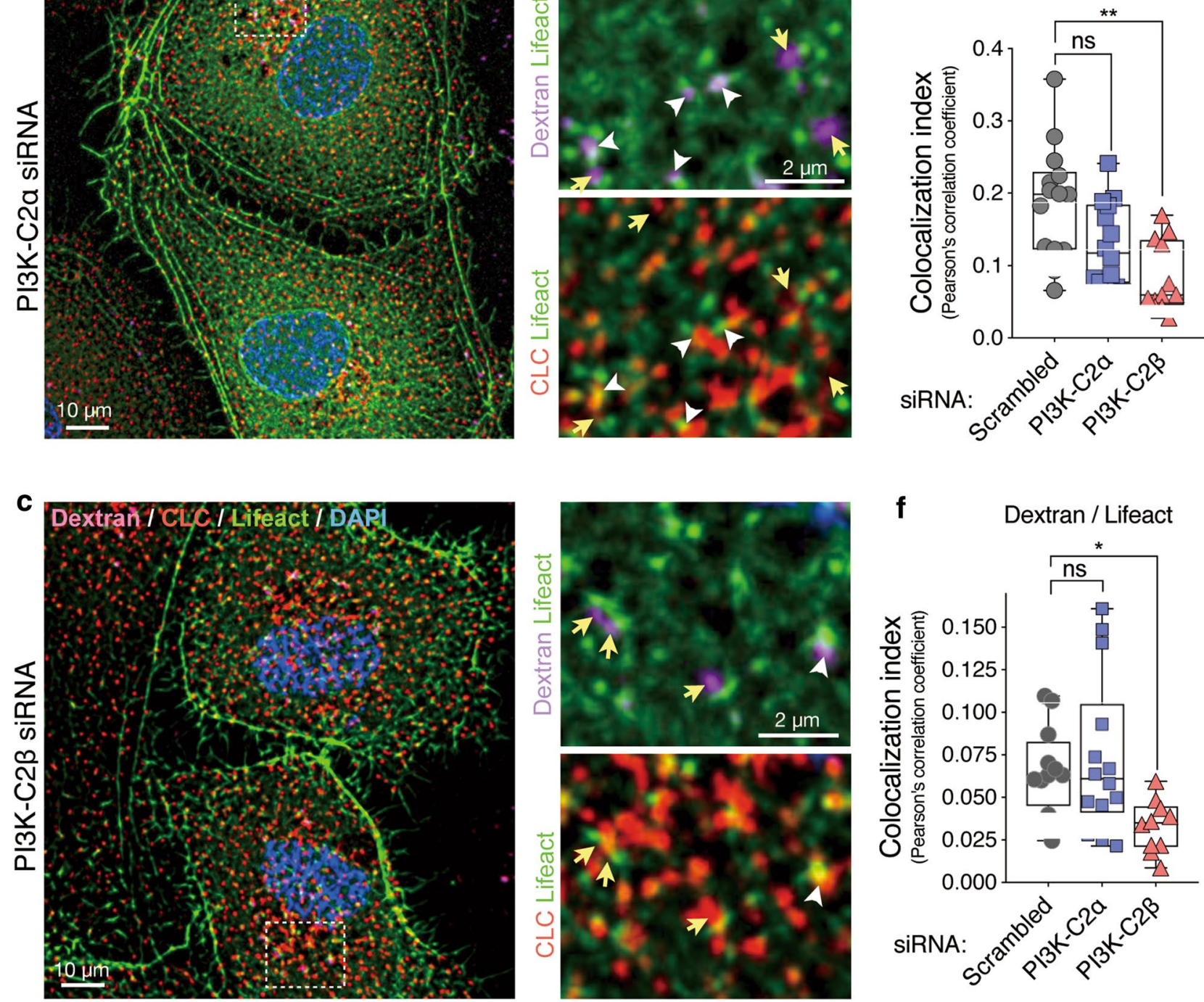

Dextran / Lifeact

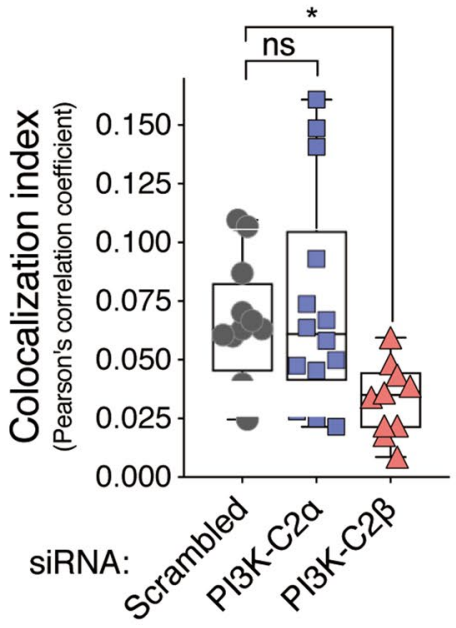


4Fig. $6 \mathrm{PI} 3 \mathrm{~K}-\mathrm{C} 2 \beta$ is required for the formation of pinosome-associated actin patches. a-c Super-resolution microscopic images of HUVECs transfected with the expression vectors of mRFP-CLC (red) and the F-actin-binding peptide mEmerald-Lifeact (green), and either of scrambled (a), PI3K-C2 $\alpha$ (b) or -C2 $\beta$ (c) siRNAs. Cells were then subjected to Alexa Fluor 647-dextran $(0.5 \mathrm{mg} / \mathrm{ml})$ uptake. Nuclei were stained with DAPI (blue). The region outlined by the dotted-box is shown at higher magnification (upper, dextran/ Lifeact; lower, CLC/Lifeact) on the right panels. White arrowheads denote clathrin-coated, dextran-containing pinosomes that are associated with actin patches, whereas yellow arrows denote clathrin-noncoated, dextran-containing pinosomes that are associated or not with actin patches. d Average number of dextran ${ }^{+}$-vesicles per cell was quantified from images. $\mathbf{e}$ and $\mathbf{f}$ Co-localization of Lifeact and CLC (e) and dextran and Lifeact (f). The co-localization indices (Pearson's correlation coefficient) are shown. Data are presented as the median and IQR in $\mathbf{d}-\mathbf{f}$. Asterisks indicate statistical significance between the indicated groups: $* p<0.05, * * p<0.01,{ }^{* * *} p<0.001$; $n s$ not significant

with or without ITSN1 overexpression (Fig. 7c, d). These observations suggest that mCherry-C $2 \beta$ overexpression stimulated pinocytosis through the mechanisms involving GFP-ITSN1-mediated recruitment of mCherry-C2 $\beta$.

\section{Endogenous ITSN1 is required for the formation of actin patches and recruitment of PI3K-C2 $\beta$ to the clathrin-coated structures}

We determined the impact of ITSN1 knockdown on F-actin structures and translocation of endogenous PI3K-C2 $\beta$. In control cells, ITSN1 showed a punctate distribution pattern with anti-ITSN1 staining, and it was partially co-localized with actin patches and clathrin-coated structures (Fig. 8a). ITSN1 knockdown effectively reduced ITSN1 expression compared with control cells and it severely impaired the formation of F-actin structures, including actin patches and stress fibers (Fig. 8a, left). In contrast, ITSN1 knockdown did not affect the number of clathrin-coated structures (Fig. 8a, right). Interestingly, ITSN1 knockdown dramatically reduced the localization of PI3K-C $2 \beta$ at actin patches (Fig. 8b, left) and at clathrin-coated structures (Fig. 8b right). These data are consistent with the notion that endogenous ITSN1 is required for the formation of clathrinassociated actin patches and PI3K-C2 $\beta$ localization at actin filament-associated clathrin-coated structures.

\section{Discussion}

Phosphoinositide 3-kinases are recognized as crucial regulators for various membrane trafficking events, which include phagocytosis, macropinocytosis and autophagy, through PI3K class-specific multiple mechanisms [11, 30-36]. In the present study we demonstrated that, among the three different PI3K classes, pinocytosis or fluid-phase endocytosis requires class II PI3K PI3K-C $2 \alpha$ and PI3K-C $2 \beta$, but not class I p110 $\alpha$ or class III Vps34. Mechanistically, both of these two class II PI3K isoforms are required for clathrinmediated, but not clathrin-non-mediated, pinocytosis, very likely through differential mechanisms.

Previous studies, including those of our group [13, 14, 21-23], have shown that PI3K-C2 $\alpha$ is closely associated with clathrin-coated pits and vesicles through its direct interaction via its $\mathrm{N}$-terminal clathrin-binding domain and that knockdown of PI3K-C2 $\alpha$ inhibits internalization of cell surface molecules. In contrast, little is understood to date on the role of PI3K-C2 $\beta$ in the regulation of endocytosis. In our study, pinocytosis in HUVECs consisted of both clathrin-mediated and -non-mediated processes, based on the dextran uptake assays. Our data of the double knockdown experiments showed that both PI3K-C $2 \alpha$ and $\mathrm{PI} 3 \mathrm{~K}-\mathrm{C} 2 \beta$ participated in clathrin-mediated, but not -nonmediated, pinocytosis. Interestingly, because knockdown of either one of PI3K-C $2 \alpha$ and PI3K-C $2 \beta$ induced inhibition of pinocytosis to a similar extent as double knockdown of PI3K-C $2 \alpha$ and PI3K-C $2 \beta$, the results suggest that $\mathrm{PI} 3 \mathrm{~K}-\mathrm{C} 2 \alpha$ and PI3K-C $2 \beta$ play distinct, non-redundant roles in clathrin-mediated pinocytosis.

A previous study [37] showed that mitotic spindle assembly required PI3K-C $2 \alpha$ protein but not its kinase activity, suggesting a scaffold role of PI3K-C $2 \alpha$ in mitotic spindle assembly. PI3K-C $2 \alpha$ has been observed to be clearly less sensitive to the pan-PI3K inhibitors WMN and LY294002, and higher concentrations of both inhibitors were required for effective inhibition of PI3K-C $2 \alpha$ compared with the other PI3K members $[38,39]$. PI3K-C2 $\beta$ was more sensitive than PI3K-C2 $\alpha$ to the PI3K inhibitors, but it was a little less sensitive to the inhibitors than class I and III PI3Ks. In the present study, only the higher concentration of WMN, but not its lower concentration, inhibited pinocytosis. Based on previous reports [38, 39], the lower concentration of WMN was expected to effectively inhibit class I and III PI3K but not PI3K-C $2 \alpha$. It is difficult to conclude whether PI3K-C2 $\beta$ was effectively inhibited or not by the lower dose of WMN. Therefore, these results could imply that the kinase activity of at least either one of PI3K-C $2 \alpha$ and PI3K-C $2 \beta$ is required for pinocytosis. In the case when one of PI3K-C $2 \alpha$ and PI3K$\mathrm{C} 2 \beta$ acts via a kinase-independent mechanism, it could include a scaffold role [37, 40]. Further investigations are necessary to explore the possibility that PI3K-C $2 \alpha$ and PI3K-C2 $\beta$ regulate pinocytosis via kinase-independent mechanisms. 


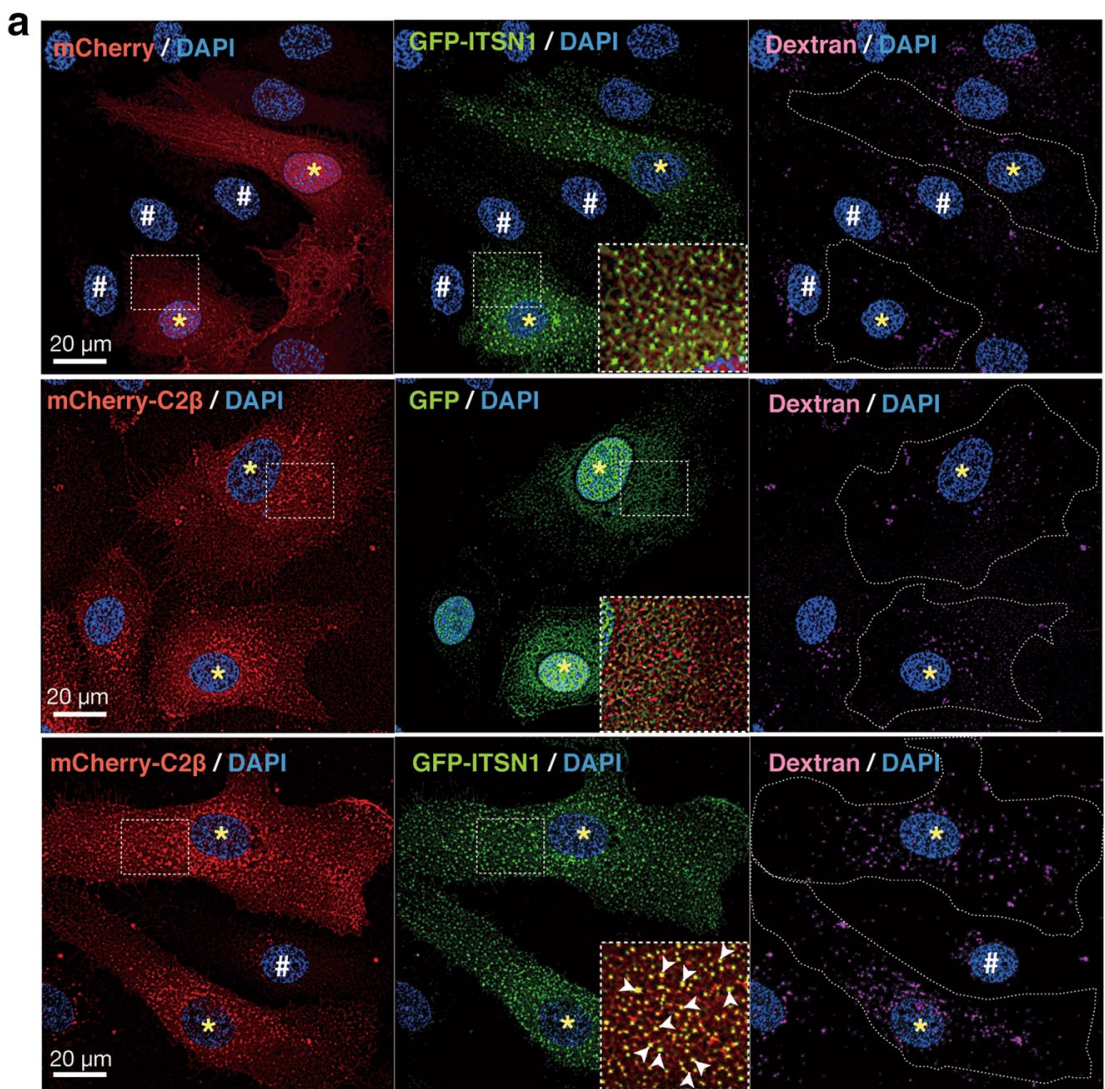

b Dextran uptake

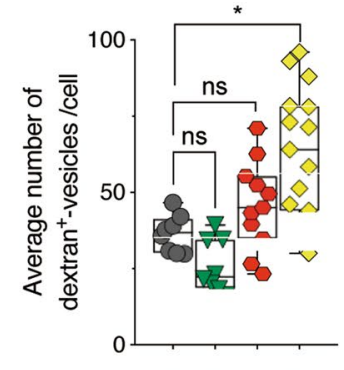

GFP

mCherry ++-

GFP-ITSN1 - + +

mCherry-C2 $\beta$ - ++

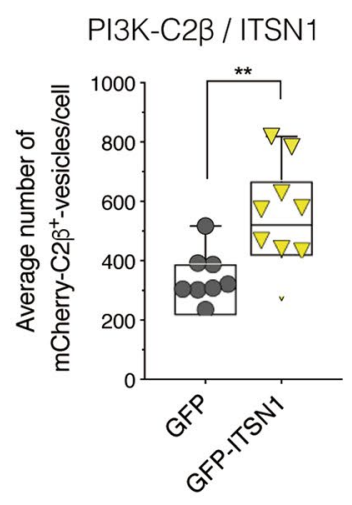

\section{c}

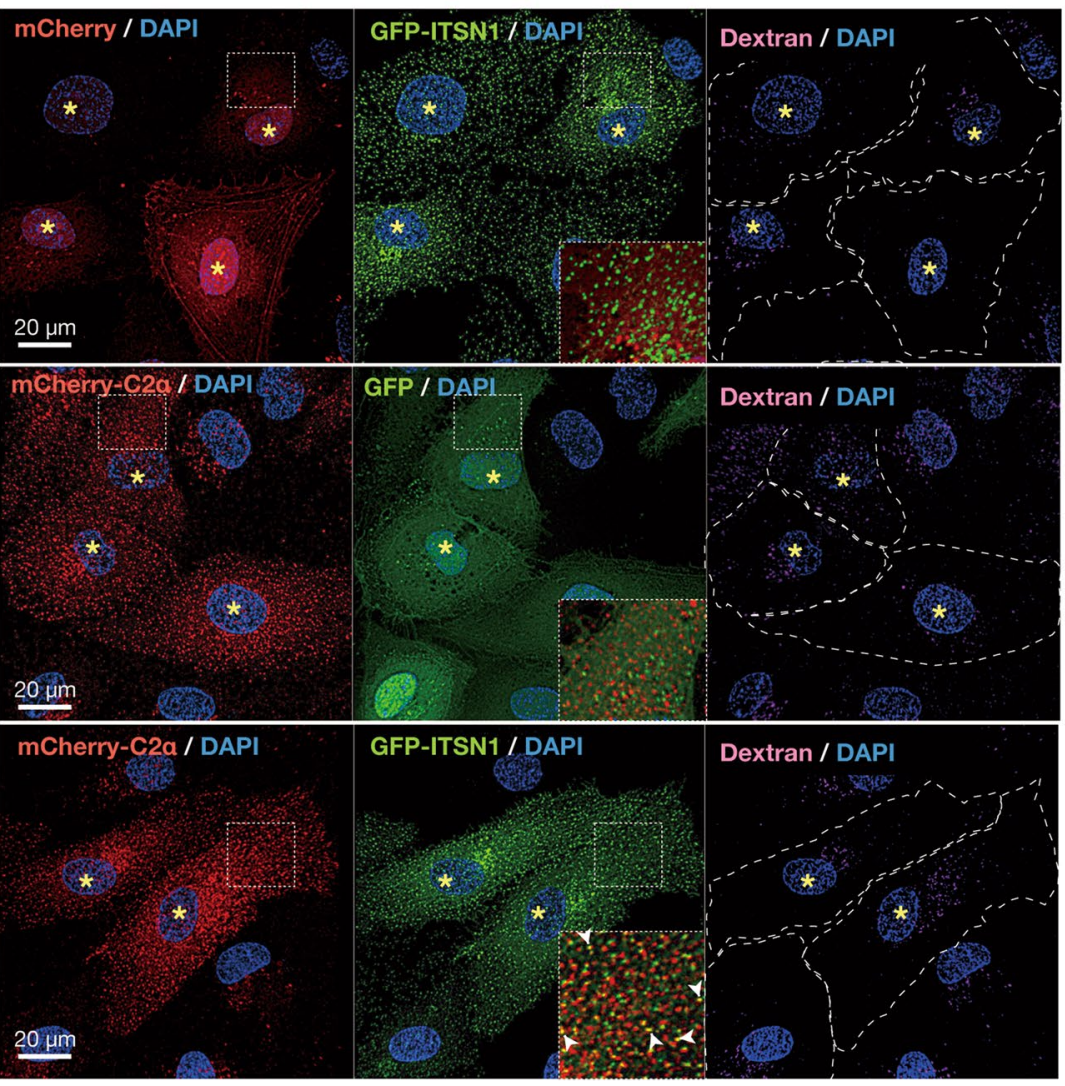

d Dextran uptake

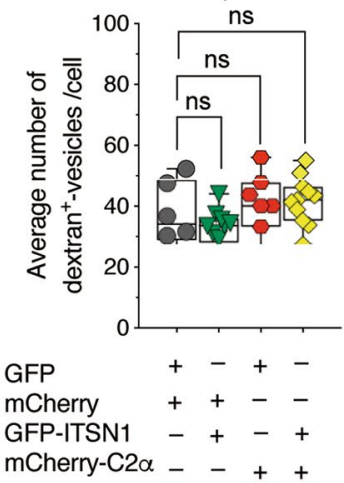

PI3K-C2 $\alpha /$ ITSN 1

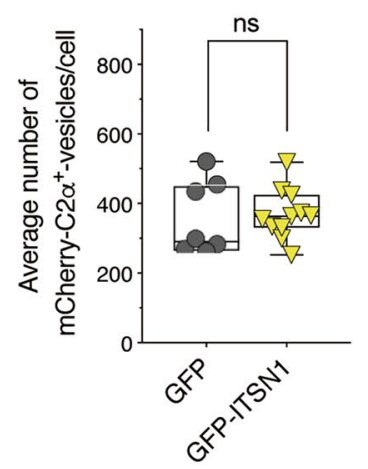


4Fig. 7 Overexpression of PI3K-C2 $\beta$ but not PI3K-C2 $\alpha$ enhances dextran uptake in the presence of intersectin-1 (ITSN1) overexpression $(\mathbf{a}, \mathbf{c})$. Super-resolution microscopic images of cells. Cells were transfected with the expression vectors of mCherry or mCherry-C2 $\beta$ or C2 $\alpha$ (red) and GFP or GFP-ITSN1 (green). The cells were then subjected to Alexa Fluor 647-dextran (purple). Nuclei were stained with DAPI (blue). Merged images of the mCherry and GFP signals in the boxed regions are shown in the lower-right panels of the middle (GFP) row, with white arrowheads (yellow-colored dots) indicating the overlap of red and green signals. Yellow asterisks indicate nuclei of vectors-transfected cells, the hashtags indicate the nuclei of nontransfected cells. b, d Upper, Average number of dextran ${ }^{+}$vesicles per cell; lower, average number of mCherry-C2 $\beta^{+}$(b) or mCherry$\mathrm{C} 2 \alpha^{+}$(d) vesicles per cell. Data are presented as the median and IQR. Asterisks indicate statistical significance between the indicated groups: ${ }^{*} p<0.05, * * p<0.01, * * * p<0.001 ; n s$ not significant

In this study, super-resolution microscopic imaging revealed three novel findings on the subcellular distribution and functions of class II PI3K in clathrin-coated pits and vesicles. First, PI3K-C $2 \beta$ and PI3K-C $2 \alpha$ were co-localized with dextran-containing, clathrin-coated endocytic vesicles (Fig. 5f). PI3K-C $2 \beta$ and PI3K-C $2 \alpha$ exhibited similar behaviors in the assembly and disassembly processes with clathrin-coated pits and/or vesicles (Fig. 5c-e). Second, the detailed microscopic analyses revealed differences in their localization at clathrin-coated pits and/or vesicles: PI3K-C $2 \beta$ showed stronger co-localization with F-actin than did PI3K-C2 $\alpha$ (Fig. 3) and was required for the actin organization at dextran-containing, clathrin-coated structures (Fig. 6c, e, f). Third, PI3K-C2 $\beta$ overexpression resulted in different effects on dextran uptake and the interaction with ITSN1 compared with PI3K-C2 $\alpha$ : the overexpression of PI3K-C $2 \beta$, but not of $\mathrm{PI} 3 \mathrm{~K}-\mathrm{C} 2 \alpha$, stimulated pinocytosis with changes in PI3KC2 $\beta$ distribution in the presence of overexpressed ITSN1.

The results of the present study and of previous studies suggest that PI3K-C $2 \alpha$ and PI3K-C $2 \beta$ are recruited to clathrin-coated pits and vesicles through different mechanisms. It is recognized that PI3K-C2 $\alpha$ is localized to clathrin-coated structures through its $\mathrm{N}$-terminal clathrin-binding domain and C-terminal PX-C2 module [22, 41]. The C-terminal PX-C2 module contributes to PI3K-C2 $\alpha$ recruitment to the clathrin-coated structures through its binding to $\mathrm{PI}(4,5) \mathrm{P}_{2}$ on the membrane. The binding of PI3K-C2 $\alpha$ to the clathrincoated membrane relieves the intramolecular autoinhibition and enhances its enzymatic activity, resulting in $\mathrm{PI}(3,4)$ $\mathrm{P}_{2}$ accumulation [41]. Accumulation of $\mathrm{PI}(3,4) \mathrm{P}_{2}$ enables recruitment of the $\mathrm{PI}(3,4) \mathrm{P}_{2}$-binding endocytic effector proteins, including SNX9, at the clathrin-coated pits to promote endocytosis. In contrast, PI3K-C $2 \beta$, which lacks the typical clathrin-binding domain, likely requires a different mechanism for its recruitment to clathrin-coated structures. A complex protein machinery which acts in the endocytic site includes ITSN1, a multifunctional scaffold protein [28]. Importantly, ITSN1 was previously identified as a binding partner of PI3K-C2 $\beta$ through the interaction between its SH3 domain and proline-rich region of PI3K-C2 $\beta$ [29]. Consistent with this observation, our study showed that ITSN1 overexpression changed the subcellular distribution of PI3K-C2 $\beta$, resulting in PI3K-C2 $\beta$ distribution as many dots. Vice versa, knockdown of ITSN1 resulted in decreased translocation of PI3K-C2 $\beta$ into clathrin-coated structures. In contrast, PI3K-C $2 \alpha$ does not possess such a proline-rich region. Therefore, ITSN1 likely does not serve as a binding partner for PI3K-C2 $\alpha$. In fact, in the present study ITSN1 overexpression did not affect the subcellular distribution of PI3K-C2 $\alpha$. A recent study [28] showed that in the endocytic site, ITSN1 also recruits the F-BAR domain-containing protein FCHSD2, which stimulates actin polymerization via the activation of WASP family proteins, resulting in the formation of actin patches around the clathrin-coated pits. The actin cytoskeleton is involved in multiple steps of clathrincoated vesicle formation, i.e. nucleation for plasma membrane invagination, progression to form mature clathrincoated pits and scission to expel vesicles into the cytoplasm, thus facilitating endocytosis [42-44]. The recruitment of the F-BAR protein FCHSD2 to the clathrin-coated pits and vesicles is a key event in clathrin-coated vesicle formation and relies on the interaction between FCHSD2 and ITSN1 and the binding of FCHSD2 to $\mathrm{PI}(3,4) \mathrm{P}_{2}$ on the membrane. Therefore, PI3K-C2 $\beta$ may contribute to actin polymerization in the endocytic site through generating $\mathrm{PI}(3,4) \mathrm{P}_{2}$ and recruiting FCHSD2. PI3K-C $2 \alpha$ and PI3K-C $2 \beta$ are both large multi-domain proteins and undergo complex interactions with multiple proteins in the clathrin-coated pits and vesicles. Therefore, although both PI3K mainly generate the same lipid product $\mathrm{PI}(3,4) \mathrm{P}_{2}$, the functional roles that $\mathrm{PI} 3 \mathrm{~K}-\mathrm{C} 2 \alpha$ and PI3K-C2 $\beta$ play in clathrin-coated vesicle formation are different.

In conclusion, the results of our study indicate that bothPI3K-C $2 \beta$ and PI3K-C $2 \alpha$ are required for fluid-phase endocytosis. Our data collectively suggest that both PI3K$\mathrm{C} 2 \alpha$ and PI3K-C2 $\beta$ participate in the clathrin-coated vesicle formation but through different mechanisms. PI3K-C $2 \beta$ promotes clathrin-coated vesicle formation very likely through stimulating F-actin formation in the endocytic site. Thus, our study emphasizes the involvement of two isoforms of class II PI3K in clathrin-mediated endocytosis. 

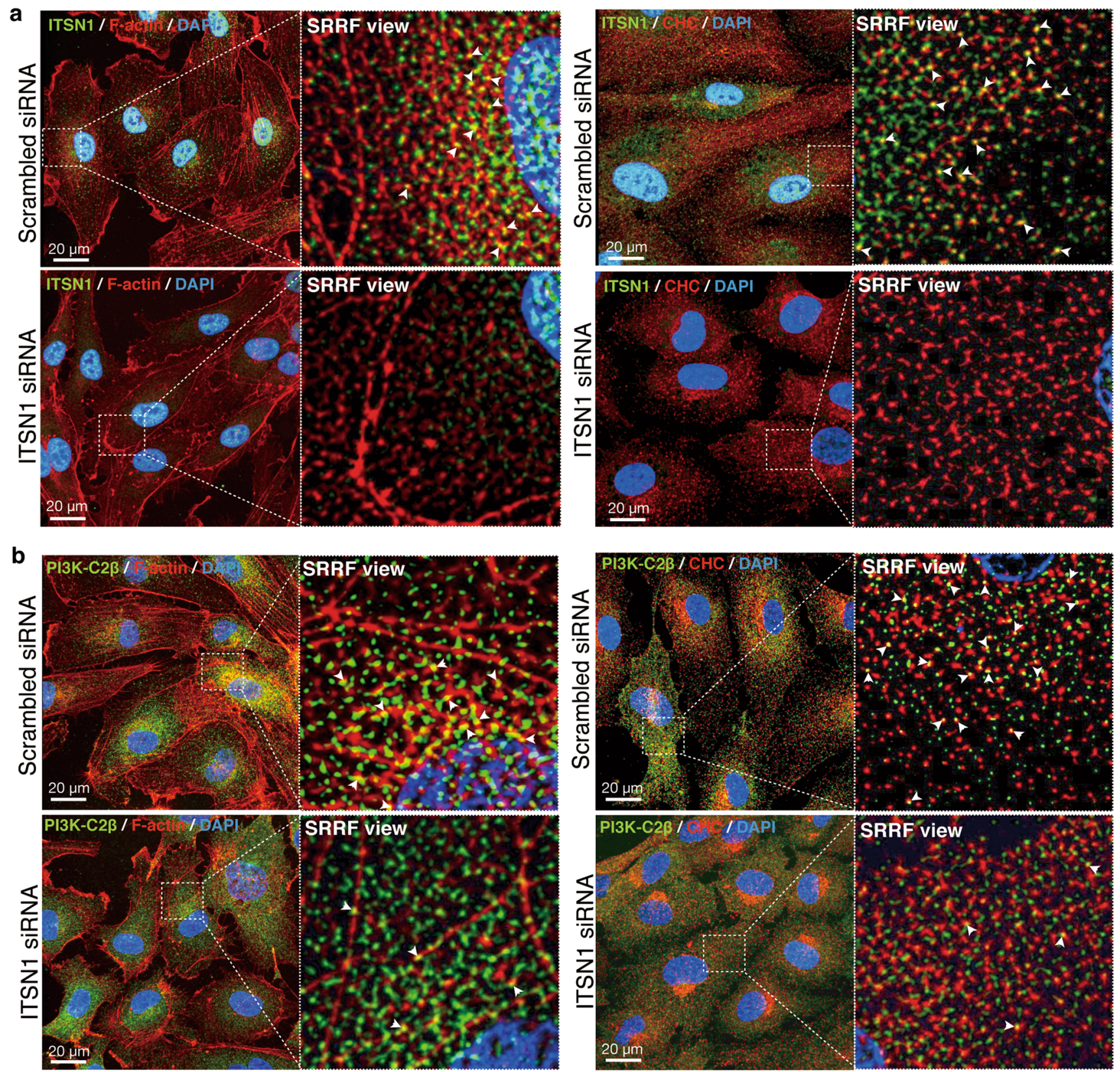

c

PI3K-C2 $\beta$ / F-actin

d

PI3K-C2 $\beta$ / CHC

4Fig. 8 Intersectin-1 is required for the formation of actin patches and the recruitment of PI3K-C2 $\beta$ to the clathrin-coated structures. a, b Confocal microscopy coupled with SRRF-Stream mode images of cells transfected with scrambled and ITSN1-specific siRNA. Double immunostaining with anti-ITSN1 (green) (a) or anti-PI3K-C2 $\beta$ (green) (b) antibodies and with Alexa Fluor 594-conjugated phalloidin (red, left panels) or anti-CHC antibody (red, right panels). Nuclei were stained with DAPI (blue). The region outlined by the dotted box is shown at higher magnification. White arrowheads in SRRF view of the merged image indicate the co-localization of endogenous ITSN1 (a) or PI3K-C2 $\beta$ (b) with F-actin or clathrin-coated structures. c, d Co-localization index of PI3K-C2 $\beta$ and F-actin (c) or CHC (d). Data are presented as the median and IQR. Asterisks indicate statistical significance between the indicated groups: $* p<0.05$, $* *<<0.01$, $* * * p<0.001$

Acknowledgements We thank Ms. Chiemi Hirose for secretarial assistance, and also thank the members of Department of Physiology for their support and assistance.

Author contributions KTA, KY, SA, and YT designed the study. KTA, $\mathrm{KY}$, and SA performed experiments. KI and NT helped experiments. KTA, KY, SA, and YT analyzed the data. KTA, KY, and YT wrote the manuscript. NT helped to draft the manuscript. All authors approved the final version of the manuscript.

Funding This study was supported by grants from the Japan International Cooperation Agency (JICA, J-14-10306 to KTA), the Ministry of Education, Culture, Sports, Science and Technology (MEXT) of Japan (25116711 to Y.T.), and the Japan Society for the Promotion of Science (17K08532 to K.Y., 16K18988 to S.A., 16K15409 to K.I., $17 \mathrm{~K} 08542$ to N.T., $15 \mathrm{H} 04673$ to Y.T.). The funders had no role in study design, data collection and analysis, decision to publish, or preparation of the manuscript.

\section{Compliance with ethical standards}

Conflict of interest The authors declare that they have no conflict of interest.

Ethical approval This article does not contain any studies with human participants or animals performed by any of the authors.

\section{References}

1. Conner SD, Schmid SL (2003) Regulated portals of entry into the cell. Nature 422:37-44. https://doi.org/10.1038/nature01451

2. Doherty GJ, McMahon HT (2009) Mechanisms of endocytosis. Annu Rev Biochem 78:857-902. https://doi.org/10.1146/annur ev.biochem.78.081307.110540

3. Kaksonen M, Roux A (2018) Mechanisms of clathrin-mediated endocytosis. Nat Rev Mol Cell Biol 19:313-326. https://doi. org/10.1038/nrm.2017.132

4. Simionescu M, Gafencu A, Antohe F (2002) Transcytosis of plasma macromolecules in endothelial cells: a cell biological survey. Microsc Res Tech 57:269-288. https://doi.org/10.1002/ jemt. 100865

5. Bohdanowicz M, Grinstein S (2013) Role of phospholipids in endocytosis, phagocytosis, and macropinocytosis. Physiol Rev 93:69-106. https://doi.org/10.1152/physrev.00002.2012
6. Hutagalung AH, Novick PJ (2011) Role of Rab GTPases in membrane traffic and cell physiology. Physiol Rev 91:119-149. https ://doi.org/10.1152/physrev.00059.2009

7. Merrifield CJ (2004) Seeing is believing: imaging actin dynamics at single sites of endocytosis. Trends Cell Biol 14:352-358. https ://doi.org/10.1016/j.tcb.2004.05.008

8. Hawkins PT, Anderson KE, Davidson K, Stephens LR (2006) Signalling through class I PI3Ks in mammalian cells. Biochem Soc Trans 34:647-662. https://doi.org/10.1042/BST0340647

9. Vanhaesebroeck B, Guillermet-Guibert J, Graupera M, Bilanges B (2010) The emerging mechanisms of isoform-specific PI3K signalling. Nat Rev Mol Cell Biol 11:329-341. https://doi. org/10.1038/nrm 2882

10. Jean S, Kiger AA (2014) Classes of phosphoinositide 3-kinases at a glance. J Cell Sci 127:923-928. https://doi.org/10.1242/ jcs.093773

11. Juhász G, Hill JH, Yan Y, Sass M, Baehrecke EH, Backer JM, Neufeld TP (2008) The class III PI(3)K Vps34 promotes autophagy and endocytosis but not TOR signaling in Drosophila. J Cell Biol 181:655-666. https://doi.org/10.1083/jcb.200712051

12. Backer JM (2008) The regulation and function of class III PI3K: novel roles for Vps34. Biochem J 410:1-17. https://doi. org/10.1042/BJ20071427

13. Yoshioka K, Yoshida K, Cui H, Wakayama T, Takuwa N, Okamoto Y, Du W, Qi X, Asanuma K, Sugihara K, Aki S, Miyazawa H, Biswas K, Nagakura C, Ueno M, Iseki S, Schwartz RJ, Okamoto H, Sasaki T, Matsui O, Asano M, Adams RH, Takakura N, Takuwa Y (2012) Endothelial PI3K-C2 $\alpha$, a class II PI3K, has an essential role in angiogenesis and vascular barrier function. Nat Med 18:1560-1569. https://doi.org/10.1038/nm.2928

14. Posor Y, Eichhorn-Gruenig M, Puchkov D, Schöneberg J, Ullrich A, Lampe A, Müller R, Zarbakhsh S, Gulluni F, Hirsch E, Krauss M, Schultz C, Schmoranzer J, Noé F, Haucke V (2013) Spatiotemporal control of endocytosis by phosphatidylinositol3,4-bisphosphate. Nature 499:233-237. https://doi.org/10.1038/ nature 12360

15. Biswas K, Yoshioka K, Asanuma K, Okamoto Y, Takuwa N, Sasaki T, Takuwa Y (2013) Essential role of class II phosphatidylinositol-3-kinase-C2 $\alpha$ in sphingosine 1-phosphate receptor-1-mediated signaling and migration in endothelial cells. J Biol Chem 288:2325-2339. https://doi.org/10.1074/ jbc.M112.409656

16. Aki S, Yoshioka K, Okamoto Y, Takuwa N, Takuwa Y (2015) Phosphatidylinositol 3-kinase class II $\alpha$-isoform PI3K-C2 $\alpha$ is required for transforming growth factor $\beta$-induced smad signaling in endothelial cells. J Biol Chem 290:6086-6105. https:// doi.org/10.1074/jbc.M114.601484

17. Marat AL, Wallroth A, Lo WT, Müller R, Norata GD, Falasca M, Schultz C, Haucke V (2017) mTORC1 activity repression by late endosomal phosphatidylinositol 3,4-bisphosphate. Science 356:968-972. https://doi.org/10.1126/science.aaf8310

18. Rozycka M, Lu YJ, Brown RA, Lau MR, Shipley JM, Fry MJ (1998) cDNA cloning of a third human C2-domain-containing class II phosphoinositide 3-kinase, PI3K-C $2 \gamma$, and chromosomal assignment of this gene (PIK3C2G) to $12 \mathrm{p} 12$. Genomics 54:569-574. https://doi.org/10.1006/geno.1998.5621

19. Falasca M, Maffucci $T$ (2012) Regulation and cellular functions of class II phosphoinositide 3-kinases. Biochem J 443:587-601. https://doi.org/10.1042/BJ20120008

20. Kitatani K, Usui T, Sriaman SK, Toyoshima M, Ishibashi M, Shigeta S, Nagase S, Sakamoto M, Ogiso H, Okazaki T, Hannun YA, Torchilin VP, Yaegashi N (2016) Ceramide limits phosphatidylinositol-3-kinase $\mathrm{C} 2 \beta$-controlled cell motility in ovarian cancer: potential of ceramide as a metastasis-suppressor lipid. Oncogene 35:2801-2812. https://doi.org/10.1038/ onc. 2015.330 
21. Domin J, Gaidarov I, Smith ME, Keen JH, Waterfield MD (2000) The class II phosphoinositide 3-kinase PI3K-C2 $\alpha$ is concentrated in the trans-Golgi network and present in clathrincoated vesicles. J Biol Chem 275(16):11943-11950. https://doi. org/10.1074/jbc.275.16.11943

22. Gaidarov I, Smith ME, Domin J, Keen JH (2001) The class II phosphoinositide 3-kinase $\mathrm{C} 2 \alpha$ is activated by clathrin and regulates clathrin-mediated membrane trafficking. Mol Cell 7:443-449. https://doi.org/10.1016/S1097-2765(01)00191-5

23. Zhao Y, Gaidarov I, Keen JH (2007) Phosphoinositide 3-kinase $\mathrm{C} 2 \alpha$ links clathrin to microtubule-dependent movement. J Biol Chem 282:1249-1256. https://doi.org/10.1074/jbc.M606998200

24. Merrifield CJ, Qualmann B, Kessels MM, Almers W (2004) Neural Wiskott Aldrich Syndrome Protein (N-WASP) and the Arp $2 / 3$ complex are recruited to sites of clathrin-mediated endocytosis in cultured fibroblasts. Eur J Cell Biol 83:13-18. https://doi. org/10.1078/0171-9335-00356

25. Benesch S, Polo S, Lai FP, Anderson KI, Stradal TE, Wehland J, Rotter K (2005) N-WASP deficiency impairs EGF internalization and actin assembly at clathrin-coated pits. J Cell Sci 118:31033115. https://doi.org/10.1242/jcs.02444

26. Galovic M, Xu D, Areces LB, van der Kammen Innocenti M (2011) Interplay between N-WASP and CK2 optimizes clathrinmediated endocytosis of EGFR. J Cell Sci 124:2001-2012. https ://doi.org/10.1242/jcs.081182

27. Hussain NK, Yamabhai M, Ramjaun AR, Guy AM, Baranes D, O’Bran JP, Der CJ, Kay BK, McPherson PS (1999) Splice variants of intersectin are components of the endocytic machinery in neurons and nonneuronal cells. J Biol Chem 274:15671-15677. https://doi.org/10.1074/jbc.274.22.15671

28. Almeida-Souza L, Frank RAW, García-Nafría J, Colussi A, Gunawardana N, Johson CM, Yu M, Howard G, Andrews B, Vallis Y, McMahon HT (2018) A flat BAR protein promotes actin polymerization at the base of clathrin-coated pits. Cell 174:325-337. https://doi.org/10.1016/j.cell.2018.05.020

29. Das M, Scappini E, Martin NP, Wong KA, Dunn S, Chen YJ, Miller SL, Domin J, O'Bran JP (2007) Regulation of neuron survival through an intersectin-phosphoinositide 3'-kinase C2 $\beta$-AKT pathway. Mol Cell Biol 27:7906-7917. https://doi.org/10.1128/ MCB.01369-07

30. Leverrier Y, Okkenhaug K, Sawyer C, Bilancio A, Vanhaesebroeck B, Ridley AJ (2003) Class I phosphoinositide 3-kinase p1 $10 \beta$ is required for apoptotic cell and Fc $\gamma$ receptor-mediated phagocytosis by macrophages. J Biol Chem 278:38437-38442. https://doi.org/10.1074/jbc.M306649200

31. Tamura N, Hazeki K, Okazaki N, Kametani Y, Murakami H, Takaba Y, Ishikawa Y, Nigorikawa K, Hazeki O (2009) Specific role of phosphoinositide 3-kinase p110 $\alpha$ in the regulation of phagocytosis and pinocytosis in macrophages. Biochem J 423:99-108. https://doi.org/10.1042/BJ20090687

32. Lim JP, Gleeson PA (2011) Macropinocytosis: an endocytic pathway for internalising large gulps. Immunol Cell Biol 89:836-843. https://doi.org/10.1038/icb.2011.20

33. Araki N, Johnson MT, Swanson JA (2012) A role for phosphoinositide 3-kinase in the completion of macropinocytosis and phagocytosis by macrophages. J Cell Biol 135:1249-1260. https ://doi.org/10.1083/jcb.135.5.1249

34. Hoeller O, Bolourani P, Clark J, Stephen LR, Hawkins PT, Weiner OD, Weeks G, Kay RR (2013) Two distinct functions for PI3kinases in macropinocytosis. J Cell Sci 126:4296-4307. https:// doi.org/10.1242/jcs. 134015

35. Nemazanyy I, Montagnac G, Russell RC, Morzygold L, Burnol AF, Guan KL, Pende M, Panasyuk G (2015) Class III PI3K regulates organismal glucose homeostasis by providing negative feedback on hepatic insulin signalling. Nat Commun 6:8283. https:// doi.org/10.1038/ncomms9283

36. Law F, Seo JH, Wang Z, Deleon JL, Bolis Y, Brown A, Zong WX, Du G, Rocheleau CE (2017) The VPS34 PI3K negatively regulates RAB-5 during endosome maturation. J Cell Sci 130:20072017. https://doi.org/10.1242/jcs. 194746

37. Gulluni F, Martini M, De Santis MC, Campa CC, Ghigo A, Margaria JP, Ciraolo E, Franco I, Ala U, Annaratone L, Disalvatore D, Bertalot G, Viale G, Noatynska A, Compagno M, Sigismund S, Montemurro F, Thelen M, Fan F, Meraldi P, Marchio C, Pece S, Sapino A, Chiarle R, Di Fiore PP, Hirsch E (2017) Mitotic spindle assembly and genomic stability in breast cancer require PI3K-C2 $\alpha$ scaffolding function. Cancer Cell 32:444-459. https:// doi.org/10.1016/j.ccell.2017.09.002

38. Domin J, Pages F, Volinia S, Rittenhouse SE, Zvelebil MJ, Stein RC, Waterfield MD (1997) Cloning of a human phosphoinositide 3-kinase with a $\mathrm{C} 2$ domain that displays reduced sensitivity to the inhibitor wortmannin. Biochem J 326(Pt 1):139-147. https://doi. org/10.1042/bj3260139

39. Stein RC, Waterfield MD (2000) PI3-kinase inhibition: a target for drug development? Mol Med Today 6:347-357. https://doi. org/10.1016/S1357-4310(00)01770-6

40. Katso RM, Pardo OE, Palamidessi A, Franz CM, Marinov M, De Laurentiis A, Downward J, Scita G, Ridley AJ, Waterfield MD, Arcaro A (2006) Phosphoinositide 3-Kinase C2 beta regulates cytoskeletal organization and cell migration via Racdependent mechanisms. Mol Biol Cell 17:3729-3744. https://doi. org/10.1091/mbc.e05-11-1083

41. Wang H, Lo WT, Vujičić Žagar A, Gulluni F, Lehmann M, Scapozza L, Haucke V, Vadas O (2018) Autoregulation of class II alpha PI3K activity by its lipid-binding PX-C2 domain module. Mol Cell 71:343-351. https://doi.org/10.1016/j.molce 1.2018.06.042

42. Merrifield CJ, Feldman ME, Wan L, Almers W (2002) Imaging actin and dynamin recruitment during invagination of single clathrin-coated pits. Nat Cell Biol 4:691-698. https://doi.org/10.1038/ ncb837

43. Yarar D, Waterman- Storer CM, Schmid SL (2005) A dynamic actin cytoskeleton functions at multiple stages of clathrinmediated endocytosis. Mol Biol Cell 16:964-975. https://doi. org/10.1091/mbc.e04-09-0774

44. Kaksonen M, Toret CP, Drubin DG (2006) Harnessing actin dynamics for clathrin-mediated endocytosis. Nat Rev Mol Cell Biol 7:404-414. https://doi.org/10.1038/nrm1940 